COMMUNICATIONS IN

ANALYSIS AND GEOMETRY

Volume 6, Number 4, 749-798, 1998

\title{
Generalizations of the Bonatti-Langevin example of Anosov flow and their classification up to topological equivalence
}

\author{
THIERRY BARBot
}

\begin{abstract}
We give a complete classification of the Anosov flows on certain 3manifolds up to topological equivalence. These manifolds are the orientable ones obtained by glueing the non-trivial circle bundle over the two-punctured projective plane along its two boundary components. We show in particular that most of these manifolds those which are not circle bundles - admit a non R-covered Anosov flow transverse to a torus. These particular Anosov flows are natural generalizations of the Bonatti-Langevin's example, and are obtained by Goodman's surgeries performed on this example. By the way, we get the first known examples of 3-manifold admitting at the same time $\mathbf{R}$-covered and non $\mathbf{R}$-covered Anosov flows.
\end{abstract}

\section{Introduction.}

We consider the problem of topological equivalence between Anosov flows (see the preliminaries for the definitions). How much does interfer the topological properties of the ambient manifold on the dynamical properties of the flow? If we consider the analogous problem for diffeomorphisms, we get a good answer, which even work in higher dimensions. This is the theorem of J. Franks: every Anosov diffeomorphism whose stable foliation is of codimension one is topologically conjugate to a linear hyperbolic diffeomorphism of a torus $([11,21])$.

But the problem for Anosov flows is deeply more intricate, even in dimension 3. The incarnation of Frank's theorem in this context is the theorem of J.F. Plante: every Anosov flow whose weak stable foliation is of codimension one on a manifold whose fundamental group is solvable ${ }^{1}$ is topologically e-

\footnotetext{
${ }^{1}$ This theorem can be improved (see [2]): it's enough to assume that the fundamental group admit a normal abelian non-cyclic subgroup. It implies for example that a torus fibered bundle over a non-solvable manifold cannot support an Anosov flow of codimension one.
} 
quivalent to the suspension of a linear hyperbolic diffeomorphism of a torus (see [23] and the remarks of [18] or of [2]). In another direction, E. Ghys showed that every Anosov flow on a circle bundle over a surface is topologically equivalent, up to finite coverings, to the geodesic flow of a hyperbolic riemannian surface ([13]). His proof can be easily extended to Anosov flows on 3-manifolds whose fundamental groups have non-trivial center ([5]).

All these (well-known) theorems are dealing with algebraic Anosov flows, i.e. roughly speaking, with left quotients by discrete subgroups of right actions on Lie groups of one-parameter subgroups (see [27]). In a preceding work ([5], theorem C) we presented other examples of Anosov flows that are completely characterized by the topology of the ambient manifold, although they are not algebraic. These examples take place in the context of graphmanifolds (see $[28,29]$ ), i.e. 3-manifolds decomposable along incompressible tori in seifert fibered spaces. The Anosov flows considered in this previous work did not admit transverse tori ${ }^{2}$.

The purpose of the present paper is to extend these results to the case of Anosov flows on graphmanifolds admitting tranverse tori. A typical illustration of this phenomena is the example of C. Bonatti and R.Langevin (see [6]). Let's give a brief description of this flow: let $\Sigma$ be the two-punctured real projective plane. Let $N$ be the total space of the non-trivial circle bundle over $\Sigma$ : it's an orientable 3-manifold containing two tori $T_{1}$ and $T_{2}$ in its boundary. On this manifold, C. Bonatti and R. Langevin have constructed an oriented foliation $\Phi_{0}$ of dimension one, transverse to the boundary and satisfying the following properties (cf. [6]):

- $\Phi_{0}$ admits one and only one periodic leaf (i.e. homeomorphic to the circle). We denote by $\theta_{0}$ this leaf.

- There are two vertical annuli $A^{s}$ and $A^{u}$ embedded in $N$ saturated by $\Phi_{0}$, whose intersection is exactly $\theta_{0}$, and such that the boundary of $A^{s}$ (respectively $A^{u}$ ) is contained in $T_{1}$ (respectively $T_{2}$ ).

- All the leaves contained in $A^{s}$ except $\theta_{0}$ start from $T_{1}$ and accumulate in the future on $\theta_{0}$.

- All the leaves contained in $A^{u}$ except $\theta_{0}$ meet $T_{2}$ in the future and accumulate in the past on $\theta_{0}$.

- All the leaves outside $A^{s}$ and $A^{u}$ are going from $T_{1}$ towards $T_{2}$.

${ }^{2}$ It was not an assumption of theorem $\mathrm{C}$ of [5], but the graphmanifolds considered in this theorem had the particularity that on some of them, there was no Anosov flow admitting transverse tori. 
We call such a foliation a BL-foliation, or BL-local flow. By glueing $T_{1}$ and $T_{2}$, we obtain an oriented foliation on a closed graphmanifold. Taking any parametrization, we get what we will call a BL-flow. C. Bonatti and R. Langevin exhibited a glueing for which the BL-flow associated is Anosov. In this paper, starting from this example and using surgeries, we extend this result in the following way (section 5):

Theorem A. Every oriented 3-manifold obtained by glueing $N$ to itself along $T_{1}$ and $T_{2}$ and which is not a circle bundle is the ambient manifold of an Anosov BL-flow.

All the oriented manifolds obtained by glueing $T_{1}$ over $T_{2}$, including the ones which are circle bundles, will be called BL-manifolds. The theorem A expresses the abundance of graphmanifolds, actually, most of the BLmanifolds, supporting Anosov flows transverse to a torus. In this paper, we give the complete classification of Anosov flows on BL-manifolds up to topological equivalence.

In order to prove the theorem A, we will use Goodman's surgeries (cf [15]). In fact, the surgeries involved are not stricto sensu those written out in [15], because in Goodman's paper, the surgeries are presented as related to the choice of a periodic orbit of the Anosov flow. We will just remark here that, as it appears implicitly in [15], the periodic orbit is not the essential feature. The real starting point of the procedure is the choice of an annulus transverse to the flow, satisfying what we will call the basket's property. We will just stress in this introduction that every transverse annulus whose boundary is nowhere tangent to the weak foliations contains a subannulus satisfying these properties. As observed S. Goodman, transverse annuli of this kind are easily found in the neighborhood of a periodic orbit. But there is another way to produce transverse annuli: in our context, they are provided by the existence of a transverse torus, and most of the transverse annuli obtained by this way are not related to periodic orbits. In section 2 , we give a brief description of this surgery along transverse annuli.

The BL-manifolds not satisfying the hypothesis of theorem A are exactly the circle bundles over $U_{3}$, the non-orientable surface of genus 3 . Thanks to Ghys's theorem noted above, all the Anosov flows on them are well-known (section 4):

Proposition. Only one of the BL-manifolds which are circle bundles does support an Anosov flow: the unitary tangent bundle of $U_{3}$. Every Anosov flow on this manifold is topologically equivalent to the geodesic flow of $U_{3}$. 
Some precision is needed: by "the geodesic flow of $U_{3}$ " we mean the geodesic flow of a hyperbolic metric on $U_{3}$, i.e. a metric of constant negative curvature. This notion is coherent up to topological equivalence, since all these geodesic flows are topologically equivalent one to the other (this is due to the connectivity of the Teichmüller space). We assume from now that some hyperbolic metric on $U_{3}$ is fixed. The choice of this metric is innocuous.

Eventually, the Handel-Thurston's methods ${ }^{3}$ provide other examples of Anosov flows on BL-manifolds: these flows will be called Handel-Thurston's examples. They are obtained by a surgery along a periodic orbit of the geodesic flow which is over a simple closed geodesic of $U_{3}$ (see remark 2.6). If this surgery is non-trivial, the ambient manifold is no more a circle bundle. Therefore it verifies the hypothesis of theorem A:

Corollary. There is an infinite family of BL-manifolds such that each of them admits at least two Anosov flows, not topologically equivalent one to the other: one which is a Handel-Thurston example, and the other a BL-flow.

An Anosov flow is said $\mathbf{R}$-covered if the lifting in the universal covering of one of its weak foliations is topologically conjugate to the foliation of $\mathbf{R}^{3}$ by horizontal planes $\mathbf{R}^{2} \times\{*\}$. According to [5] a $\mathbf{R}$-covered Anosov flow cannot be transverse to a transverse torus, unless it is a suspension. On the other hand, as it is proved in [5], all the Handel-Thurston examples are $\mathbf{R}$-covered. Thus, the corollary provides the first known examples of 3 manifolds admitting at the same time $\mathbf{R}$-covered and non $\mathbf{R}$-covered Anosov flows.

At this step, we completely understood what are the BL-manifolds supporting Anosov flows. We are left with the problem of the classification of the Anosov flows up to topological equivalence on each of them. We will prove that the list above exhausts all the possibilities:

Theorem B. Let $M$ be a BL-manifold.

(1) If $M$ is a circle bundle, then it supports an Anosov flow if and only if it's diffeomorphic to the unitary fibered tangent space of the surface $U_{3}$. In this last case, every Anosov flow on $M$ is topologically equivalent to the geodesic flow of $U_{3}$.

\footnotetext{
${ }^{3}$ Which is nothing but a particular application of Goodman's surgery. The paper [15] is in fact directly inspired by the construction of Handel-Thurston [16].
} 
(2) If $M$ is not a circle bundle, then it admits at least one non $\mathbf{R}$-covered Anosov flow. All the non $\mathbf{R}$-covered Anosov flow on $M$ are BL-flows and are topologically equivalent one to the other.

(3) If $M$ is not a circle bundle, the $\mathbf{R}$-covered Anosov flows on $M$, if they exist, are all topologically equivalent to an example of HandelThurston.

Furthermore, all these Anosov flows are topologically equivalent to volume preserving flows.

The first point of theorem B is exactly the proposition above. The third point is proved at section 4 . Note the anecdotic fact that the ambient manifold of the Bonatti-Langevin's example is not one of the manifolds satisfying the third point of theorem B. Note also that the BL-manifolds admitting examples of Handel-Thurston are easily characterized. Unfortunately, there is no elegant or concise criterion distinguishing them from the others.

The second point of theorem B is proved at the section 9. It's certainly the most original result of this paper. Its proof can be sum up as follow: we first show that every non R-covered Anosov flow on a BL-manifold is transverse to a torus (theorem 7.3). We denote by $T$ this transverse torus. Cutting along $T$, we obtain an oriented one-dimensional foliation $\Phi_{1}$ on $N$. The weak foliations of the flow give rise in $N$ to two foliations of codimension one, transverse one to the other and transverse to the boundary. The intersection of this two foliations is exactly $\Phi_{1}$. Studying these foliations, we prove that $\Phi_{1}$ is a BL-foliation (proposition 8.5). In particular, $\Phi_{1}$ preserves two vertical annuli $A^{s}$ and $A^{u}$. Since the topology of the BL-manifold $M$ is quite simple, the triple $\left(T, A^{s}, A^{u}\right)$ does not depend up to isotopy to the initial Anosov flow: if we fix two non $\mathbf{R}$-covered Anosov flows on $M$, we can always assume after isotopy that they are associated to the same triple. Thus, to show that they are topologically equivalent is equivalent to show that $\left(T, A^{s}, A^{u}\right)$ characterizes the Anosov flow up to topological equivalence. This plan is fulfilled at section 9 in the following manner: we associate to $\left(T, A^{s}, A^{u}\right)$ a combinatorial dynamical system which encodes the itineraries of the orbits of the flow whose intersection with $T$ is infinite. Its definition looks like the definition of the Bernouilli system associated to a Markov partition (see [7]). The crucial point is that this combinatorial dynamical system can be defined only using the topological properties of $M$, independently of the flow. We then conclude by checking that the knowledge of the restriction of the Anosov flow to the set of the orbits meeting an infinite number of time the torus $T$ is enough for recovering the whole Anosov flow. 
I would like to thank J.M. Lion and C. Bonatti for stimulating conversations. The idea of this paper emerged when C. Bonatti reported me a lecture of P. Foulon dealing with the interpretation of Goodman's surgery reproduced at section 2 of the present paper.

\section{Preliminaries.}

Let $M$ be a closed orientable 3-manifold. Let $\Phi^{t}$ be a flow on $M$ generated by a vector field of class $C^{r}(r \geq 1)$. We denote by $\Phi$ the oriented foliation whose leaves are the orbits of $\Phi^{t}$. Two flows are topologically equivalent if the corresponding oriented foliations are topologically conjugate. The flow $\Phi^{t}$ is Anosov if there is a continuous decomposition of the tangent bundle $T M$ as a Whitney sum $T M=E^{0} \oplus E^{s} \oplus E^{u}$ of $D \Phi^{t}$ invariant one dimensional subbundles and there are constants $\mu_{0} \geq 1, \mu_{1}>0$ so that:

(i) $E^{0}$ is tangent to the flow,

(ii) $D \Phi^{t}(v) \leq \mu_{0} e^{-\mu_{1} t} v$ for any $v \in E^{s}, t \geq 0$,

(iii) $D \Phi^{-t}(v) \leq \mu_{0} e^{-\mu_{1} t} v$ for any $v \in E^{u}, t \geq 0$.

The line fields $E^{s}$ and $E^{u}$ are in general only continuous, but they are always uniquely integrable. They define two one-dimensional foliations $\mathcal{F}^{s s}$ and $\mathcal{F}^{u u}$ called the strong stable and strong unstable foliations of the flow. Furthermore, the plane fields $E^{0} \oplus E^{s}$ and $E^{0} \oplus E^{u}$ are also integrable, producing foliations $\mathcal{F}^{s}, \mathcal{F}^{u}$ of codimension one which are the weak stable and weak unstable foliations of the flow (for all these results see [1]). The foliation $\Phi$ is the intersection of $\mathcal{F}^{s}$ and $\mathcal{F}^{u}$. We can derive from this that the Anosov property is a property of the foliation. In other words, if one parametrization of $\Phi$ is an Anosov flow, then all the other parametrizations are Anosov flows too ([14]). An important property of Anosov flows is the structural stability: any flow of class $C^{1}$ on $M C^{1}$-near of $\Phi^{t}$ is still Anosov, and topologically equivalent to $\Phi^{t}([1])$. From this fact arise few comments:

- this shows the naturality of the problem of the classification of Anosov flows up to topological equivalence,

- since we are only concerned by the Anosov flows up to topological equivalence, by taking a smooth approximation we can always assume that the flow is smooth,

- finally, it implies that the flow preserves many differentiable structures on the topological manifold $M$, and none of them is a priori better 
than the others. In this sense, Anosov flows are topological objects. Once more, this justify the concern of relating them with the topology of the ambient manifold.

When the Anosov flow is smooth, and we can always assume it thanks to the remark above, the weak foliations are of class $C^{1}([17])$. The leaves of these foliations are either topological planes, annuli or Möbius bands. The last two correspond exactly to leaves containing closed orbits of $\Phi$. There is at most one closed orbit in a leaf of $\mathcal{F}^{s}$ in which case all other orbits are forward asymptotic to it. Similarly for $\mathcal{F}^{u}$. Both weak foliations are Reebless, so Novikov's theorem implies that none of them admits a closed transversal null homotopic. Also, no closed orbit of $\Phi$ has a null homotopic power.

The flow is topologically transitive if the non-wandering set is the whole manifold [25]. Equivalently, the periodic orbits form a dense subset of $M$, or there is a dense orbit, or every leaf of the weak foliations is dense $[1,22,25]$.

Let $\pi: \widetilde{M} \rightarrow M$ be the universal covering of $M$. The foliations $\mathcal{F}^{s s}$, $\mathcal{F}^{u u}, \mathcal{F}^{s}, \mathcal{F}^{u}$ and $\Phi$ lift to foliations $\widetilde{\mathcal{F}}^{s s}, \widetilde{\mathcal{F}}^{u u}, \widetilde{\mathcal{F}}^{s}, \widetilde{\mathcal{F}}^{u}$ and $\widetilde{\Phi}$ in $\widetilde{M}$. We denote by $\widetilde{\Phi}^{t}$ the lifting of $\Phi^{t}$. The foliation $\widetilde{\mathcal{F}}^{s}$ is a foliation by planes, so $\widetilde{M}$ is homeomorphic to $\mathbf{R}^{3}$. Let $Q^{\Phi}$ be the orbit space of $\widetilde{\Phi}$ obtained by collapsing flow lines to points, and $\pi^{\Phi}$ the projection map. This quotient is Hausdorff and hence homeomorphic to $\mathbf{R}^{2}$ [3]. We call it abusively the orbit space of $\Phi^{t}$. The map $\pi^{\Phi}$ is a trivial fibration. The foliations $\widetilde{\mathcal{F}}^{s}$ and $\widetilde{\mathcal{F}}^{u}$ induce two foliations in $Q^{\Phi}$ by lines transverse one to the other. We denote them by $\mathcal{G}^{s}$ and $\mathcal{G}^{u}$ respectively. Remark that each leaf of $\mathcal{G}^{s}$ intersects each leaf of $\mathcal{G}^{u}$ on at most one point.

Let $\Gamma$ be the fundamental group of $M$. Its action on $\widetilde{M}$ by covering translations induces an action on $Q^{\Phi}$ which preserves the two foliations $\mathcal{G}^{s}$ and $\mathcal{G}^{u}$. This action contains all the information about the flow. More precisely [3]:

Theorem 1.1. Two Anosov flows are topologically equivalent, or one is topologically equivalent to the inverse of the other, if and only if there is an homeomorphism between their orbit spaces equivariant for the respective actions of the fundamental groups.

This action is effective. Each non trivial element $\gamma$ of $\Gamma$ is $C^{1}$-linearisable in the neighborhood of each of its fixed points. Note that since we assume $M$ oriented, the orbit space is naturally oriented, and this orientation is preserved by the $\Gamma$-action. 
Let $Q^{s}$ be the quotient of $\widetilde{M}$ by the relation "being on the same leaf of $\widetilde{\mathcal{F}}^{s}$. We call it the stable leaf space of the flow. This is a connected, simply-connected one dimensional manifold but in general non Hausdorff. Similarly, we define the unstable leaf space of the flow $Q^{u}$. We denote by $\pi^{s}$ and $\pi^{u}$ the quotient maps. There are natural maps $p^{s}: Q^{\Phi} \rightarrow Q^{s}$ and $p^{u}: Q^{\Phi} \rightarrow Q^{u}$. The action of $\Gamma$ on $\widetilde{M}$ induces an action on each leaf space. The properties of the weak foliations incarnate in the properties of these actions in the following manner:

- these actions are effective,

- the isotropy group of an element of $Q^{s}$ is trivial or cyclic,

- near a fixed point, a non trivial element of $\gamma$ is a contraction or a dilatation.

Since they are simply-connected, the leaf spaces are orientable. Note that since the orbit space is oriented, an orientation on one leaf space induces an orientation on the other leaf space, and an element of $\Gamma$ which reverses the orientation of one leaf space reverses the orientation of the other. We assume from now that such an orientation has been fixed.

If one of the leaf spaces is Hausdorff, then the other is Hausdorff too (cf. [3]). Such an Anosov flow is said $\mathbf{R}$-covered. We remind the reader of some properties of the $\mathbf{R}$-covered Anosov flows non topologically equivalent to supensions [3]:

- each leaf space is homeomorphic to $\mathbf{R}$,

- such a flow is topologically equivalent to its inverse,

- there is no embedding of the torus in $M$ transverse to the flow,

- the flow is topologically transitive,

- on each leaf space, there is an homeomorphism $\tau$ without fixed points and commuting with every orientation preserving element of $\Gamma$. Moreover, for each non trivial orientation preserving element $\gamma$ of $\Gamma$, the set of the $\gamma$-fixed points is empty or is the union of two $\tau$-orbits. Finally, if $\gamma$ orientation reversing, then $\tau \circ \gamma=\gamma \circ \tau^{-1}$.

In any case, the orbit space $Q^{\Phi}$ embeds naturally in the product $Q^{s} \times Q^{u}$ of the leaf spaces: the map which associates to an orbit the leaves of $\widetilde{\mathcal{F}}^{s}$ and $\widetilde{\mathcal{F}}^{u}$ containing it is an homeomorphism on its image commuting with the 
actions of the fundamental group (the natural action of $\Gamma$ on the product is the diagonal one). We will sometimes identify abusively an element of $Q^{\Phi}$ with its image by this map. We call Markov rectangle a closed subset of $Q^{\Phi}$ image by this map of the product $I^{s} \times I^{u}$ of two intervals of $Q^{s}$ and $Q^{u}$.

For each element $x$ of $Q^{\Phi}$, we denote by $s(x)$ (respectively $u(x)$ ) the leaf of $\mathcal{G}^{s}$ (respectively of $\mathcal{G}^{u}$ ) containing $x$. The orientation of $Q^{u}$ induces an orientation, hence, an order on $s(x)$. We denote by $s^{+}(x)$ the connected component of $s(x) \backslash\{x\}$ formed by the elements greater than $x$, and $s^{-}(x)$ the other connected component. Similarly we define $u^{+}(x)$ and $u^{-}(x)$. Let $S_{+}(x)$ be the connected component of $Q^{\Phi} \backslash s(x)$ containing $u^{+}(x)$, and $S_{-}(x)$ the other one. We define analogously $U_{+}(x)$ and $U_{-}(x)$. For each element $s=s(x)$ of $Q^{s}$, we denote by $s_{+}$and $s_{-}$the images by $p^{s}$ of $S_{+}(x)$ and $S_{-}(x)$. There are the connected components of $Q^{\Phi} \backslash\{s\}$. Similarly, we denote by $u_{+}$and $u_{-}$the connected components of the complement of an element $u$ of $Q^{u}$. Finally, to each element $x$ of $Q^{\Phi}$ we associate the following four open subsets (we denote by $\operatorname{Sat}_{\mathcal{G}^{s}}\left(u^{+}(x)\right.$ ) the saturation of $u^{+}(x)$ by $\left.\mathcal{G}^{s}\right)$ :

$$
\begin{aligned}
& \mathcal{L}^{++}(x)=\operatorname{Sat}_{\mathcal{G}^{s}}\left(u^{+}(x)\right) \cap \operatorname{Sat}_{\mathcal{G}^{u}}\left(s^{+}(x)\right) \\
& \mathcal{L}^{+-}(x)=\operatorname{Sat}_{\mathcal{G}^{s}}\left(u^{+}(x)\right) \cap \operatorname{Sat}_{\mathcal{G}^{u}}\left(s^{-}(x)\right) \\
& \mathcal{L}^{-+}(x)=\operatorname{Sat}_{\mathcal{G}^{s}}\left(u^{-}(x)\right) \cap \operatorname{Sat}_{\mathcal{G}^{u}}\left(s^{+}(x)\right) \\
& \mathcal{L}^{--}(x)=\operatorname{Sat}_{\mathcal{G}^{s}}\left(u^{-}(x)\right) \cap \operatorname{Sat}_{\mathcal{G}^{u}}\left(s^{-}(x)\right)
\end{aligned}
$$

An open subset $\mathcal{L}$ of $Q^{\Phi}$ is a lozenge if there are two elements $x$ and $y$ of $Q^{\Phi}$ such that $\mathcal{L}=\mathcal{L}^{++}(x)=\mathcal{L}^{--}(y)$ or $\mathcal{L}=\mathcal{L}^{+-}(x)=\mathcal{L}^{-+}(y)$. The pair $(x, y)$ is then unique. If an element $\gamma$ fixes globally the lozenge, then $\mathcal{L}$ is a $\gamma$-lozenge. This useful notion of lozenge has been introduced by $\mathrm{S}$. Fenley [10]. As noticed in [4], these lozenges correspond in a nice way to annuli embedded in $M$, transverse to the flow except at their boundary which is the union of two periodic orbits. In a general way, let $S$ be an incompressible surface $S$ embedded in $M$, whose boundary is tangent to the flow and whose interior is transverse to the flow. The universal covering $\widetilde{S}$ of $S$ embeds naturally in $\widetilde{M}$ as a lifting of $S$. We define the shadow of $S$ as the image of $\widetilde{S}$ by $\pi^{\Phi}$. It's well-defined up to the action of $\Gamma$ on $Q^{\Phi}$. We are interested by this notion because of the following phenomenon, illustrated in [4]: roughly speaking, the problem of finding a surface isotopic to $S$ and in "good" position with respect to $\Phi^{t}$ is intimately related to the problem of finding a "good" subset of $Q^{\Phi}$ preserved by the subgroup of $\Gamma$ corresponding to the fundamental group of $S$. This "good" subset can be viewed as the shadow of the "good" position of $S$. We will use this principle in order to 
exhibit transverse tori. In our case, the shadow of the transverse torus will be the support of a s-sequence (or u-sequence) of lozenges: a sequence of lozenges $\mathcal{L}_{n}$ indexed by $\mathbb{Z}$ is a s-sequence of lozenges if there is a sequence $\left(x_{i}\right)$ of elements of $Q^{\Phi}$ so that for all integer $p$ :

$$
\begin{gathered}
\mathcal{L}_{2 p-1}=\mathcal{L}^{++}\left(x_{2 p}\right)=\mathcal{L}^{--}\left(x_{2 p-1}\right) \\
\mathcal{L}_{2 p}=\mathcal{L}^{+-}\left(x_{2 p+1}\right)=\mathcal{L}^{-+}\left(x_{2 p}\right)
\end{gathered}
$$

The union of this lozenges with the $s^{+}\left(x_{2 p}\right)$ and the $s^{-}\left(x_{2 p+1}\right)$ is an open subset of $Q^{\Phi}$. We call it the support of the s-sequence.

A sequence $\mathcal{L}_{n}$ is a u-sequence of lozenges if there is a sequence $\left(y_{i}\right)$ of elements of $Q^{\Phi}$ so that for all integer $p$ :

$$
\begin{gathered}
\mathcal{L}_{2 p-1}=\mathcal{L}^{++}\left(y_{2 p}\right)=\mathcal{L}^{--}\left(y_{2 p-1}\right) \\
\mathcal{L}_{2 p}=\mathcal{L}^{+-}\left(y_{2 p}\right)=\mathcal{L}^{-+}\left(y_{2 p+1}\right)
\end{gathered}
$$

The union of this lozenges with the $u^{+}\left(y_{2 p}\right)$ and the $u^{-}\left(x_{2 p+1}\right)$ is an open subset of $Q^{\Phi}$ called the support of the $u$-sequence. In the figure 1 we give a partial picture of a $s$-sequence and of a $u$-sequence with the same support.

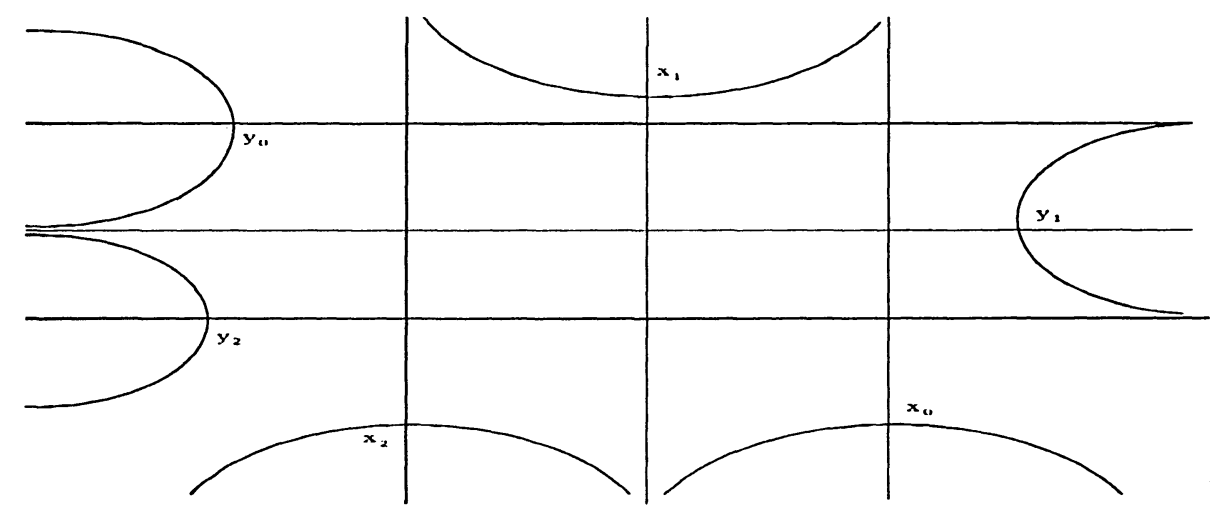

Figure 1: Partial view of the common support of a $s$-sequence and a $u$-sequence.

Another nice feature involving lozenges is the following result (see [10]): 
Proposition 1.2. Let $x$ and $y$ be two different fixed points in $Q^{\phi}$ of a non trivial element $\gamma$ of $\Gamma$. Then, there is sequence of points

$$
x_{1}=x, x_{2}, \ldots, x_{k}=y
$$

such that, for every index $i$, the points $x_{i}$ and $x_{i+1}$ are the two vertices of a $\gamma$-lozenge. This sequence is unique if we impose that the lozenges involved are pairwise disjoints.

The unique sequence of lozenges appearing in the proposition above is called the sequence of lozenges joining $x$ and $y$.

We achieve this section by generalities and notations in the context of connected simply-connected non-Hausdorff one dimensional manifolds. All the notions involved are very similar to the notions appearing in the study of the automorphisms of $\mathbf{R}$-trees (cf. [26, 19]).

Let $Q$ be such a manifold. We fix an orientation on it. If two elements $x$ and $y$ of $Q$ are not separated by the topology of $Q$, we write $x \approx y$. Such elements are called branching points. An (open) interval of $Q$ is the image of a local homeomorphism from $\mathbf{R}$ into $Q$. Two elements $x$ and $y$ of $Q$ are said comparable if they belong to the same interval. In this case, we denote by] $x, y$ [ the set of the elements of $Q$ which disconnect $x$ from $y$ : it's an open interval. We denote by $[x, y]$ the union of $] x, y[$ with $\{x, y\}$. Note that in general $[x, y]$ is not closed. The orientation of $Q$ induce an orientation on each open interval, hence, an order between comparable elements. We denote by $\prec$ this order. For each element $x$ of $Q$, we denote by $x_{+}$the connected component containing the elements $\prec$-bigger than $x$, and $x_{-}$the other connected component.

More generally, for every pair $(x, y)$ of elements of $Q$, we denote by ]$x, y[$ the set of the elements of $Q$ which disconnect $x$ and $y$. The union ]$x, y[\cup\{x, y\}$ is noted $[x, y]$. If $x$ and $y$ are comparable, this coincide with the previous definition. If not, the set $[x, y]$ is a finite union of "closed" intervals $\left[x_{i}, y_{i}\right]$ such that $y_{i} \approx x_{i+1}$.

Let $\gamma: Q \rightarrow Q$ be a homeomorphism. We assume $\gamma$ to be orientation preserving. We denote by $F i x(\gamma)$ the set of the $\gamma$-fixed points. Let $F i x \approx(\gamma)$ be the set of the elements $x$ of $Q$ such that $\gamma(x) \approx x$ : it's a closed subset containing the closure of $F i x(\gamma)$. Its elements are called quasi-fixed points. We say that $\gamma$ separates the points of $Q$ if $F i x^{\approx}(\gamma)$ is empty. In this case, we define the fundamental axis of $\gamma$ as follows: it is the set of points $x$ such that $\gamma\left(x_{+}\right)$contains $x_{+}$, or is contained in $x_{+}$.

Proposition. [2,5] The fundamental axis of a homeomorphism $\gamma$ separating the points of $Q$ is non empty. It is an open interval, or an union 
of closed intervals $\left[x_{i}, y_{i}\right]$ indexed by $\mathbb{Z}$ such that for all integer $i$ we have $y_{i} \approx x_{i+1}$. In the last case, there is an integer $k$ such that for each integer $i$, the image of $x_{i}$ and $y_{i}$ by $\gamma$ are respectively $x_{i+k}$ and $y_{i+k}$. The fundamental axis is preserved by every homeomorphism $\gamma^{\prime}$ of $Q$ commuting with $\gamma$, or conjugating $\gamma$ with its inverse.

We can deduce from this proposition that for every orientation preserving homeomorphism $\gamma$ of $Q$ and for each non trivial integer $n, \gamma$ separates the points of $Q$ if and only if $\gamma^{n}$ does.

We will need the following proposition:

Proposition 1.3. Let $\gamma_{1}$ and $\gamma_{2}$ be two homeomorphisms of $Q$. We assume that each of them admits fixed points in $Q$, and that they commute. Then, there is a fixed point of one of them which is a quasi-fixed point of the other.

Proof. If one of the homeomorphism reverses the orientation, let's say $\gamma_{1}$, then the proposition is obvious since this homeomorphism should admit a unique fixed point, and the other homeomorphism $\gamma_{2}$ should then preserve this unique fixed point. Thus, we assume that they are both orientation preserving. Let $U$ be the complement in $Q$ of $F i x^{\approx}\left(\gamma_{1}\right)$. It's an open subset of $Q$ which is preserved by $\gamma_{1}$ and $\gamma_{2}$. If $\gamma_{2}$ admits no fixed point in $U$, then it admits some fixed point in Fix $\approx\left(\gamma_{1}\right)$ and we are done. Therefore, we assume that some element $x_{2}$ of $U$ is fixed by $\gamma_{2}$. Let $U_{2}$ be the connected component of $U$ containing $x_{2}$ : it's globally preserved by $\gamma_{2}$.

Assume that $U_{2}$ is not $\gamma_{1}$-invariant. Then, $\gamma_{1} U_{2}$ is another connected component of $U$ disjoint from $U_{2}$. For each element $x$ of the frontier $\partial U_{2}$, let $x^{c}$ be the connected component of $Q \backslash\{x\}$ containing $U_{2}$. Then, $U_{2}$ is exactly the intersection of the $x^{c}$ where $x$ describes all $\partial U_{2}$. Moreover, $Q$ is the union of $U_{2}$ and of the $Q \backslash x^{c}$. Let $x$ be the unique element of $\partial U_{2}$ such that $x^{c}$ contains $\gamma_{1} U_{2}$. Then, $x$ is fixed by $\gamma_{2}$. On the other hand, it belongs to $\partial U_{2}$ which is contained in $F i x^{\approx}\left(\gamma_{1}\right)$.

The following proposition works in the context of leaf spaces of Anosov flows. Its analog in the general case is no more true (cf. $[10,4])$ :

Proposition 1.4. Let $Q$ be a leaf space associated to an Anosov flow, and $\gamma$ a non trivial element of the fundamental group of the ambient manifold. We assume that the action of $\gamma$ on $Q$ is orientation preserving. Let $n$ be a non-trivial integer. Then, every fixed point of the action of $\gamma^{n}$ on $Q$ is a fixed point of the action of $\gamma$ on $Q$. 


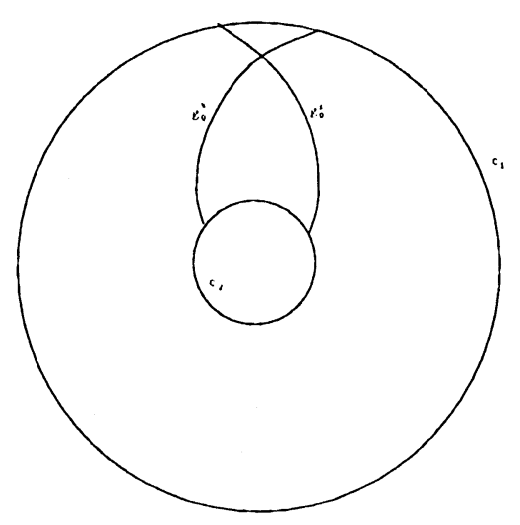

Figure 2: The natural orientation on $A$

\section{Surgeries on Anosov flows.}

Let $A$ be an open annulus embedded in $M$ and transverse to an Anosov flow $\Phi^{t}$. Then the weak foliations $\mathcal{F}^{s}$ and $\mathcal{F}^{u}$ are transverse to $A$ and define on it two foliations $g^{s}$ and $g^{u}$ of dimension one and transverse one to the other. Let $A_{0}$ be a closed sub-annulus of $A$. We denote by $g_{0}^{s}$ and $g_{0}^{u}$ the restrictions of $g^{s}$ and $g^{u}$ to $A_{0}$. We assume that they satisfy the following property, that we call the basket's property:

Each leaf of $g_{0}^{s}$ or $g_{0}^{u}$ is a closed path joining the two boundary components of $A_{0}$.

We claim that such a bifoliation induces a natural orientation of $A: c_{1}$ and $c_{2}$ be the two boundary components of $A_{0}$. We fix one of them, say $c_{1}$. Then, we parametrise $g_{0}^{s}$ and $g_{0}^{u}$ in such a way that each leaf for these orientations goes from $c_{1}$ towards $c_{2}$. This defines a basis $\left(U_{x}^{0}, S_{x}^{0}\right)$ over each point $x$ of $A_{0}$, where $U_{x}^{0}$ and $S_{x}^{0}$ are respectively tangents to $g_{0}^{s}$ and $g_{0}^{u}$, and thus an orientation of $A_{0} \subset A$. If we exchange $c_{1}$ and $c_{2}$, we inverse both parametrisations: the orientation is thus not modified.

Let $\varphi: A_{0} \rightarrow A_{0}$ be a diffeomorphism which is the identity near $\partial A_{0}$. Its isotopy class modulo $\partial A_{0}$ is a Dehn twist: since we have fixed an orientation, it's uniquely defined by an integer $n$. We blow up $M$ along $A_{0}$ : we obtain a manifold with corner $M_{*}$ whose boundary is the union of two copies $A_{1}$ and 
$A_{2}$ of $A_{0}$ sharing the same boundary. The flow $\Phi^{t}$ corresponds to a local flow $\Phi_{*}$ on $M_{*}$ transverse to $A_{1}$ and $A_{2}$. We choose the notations such that $\Phi_{*}$ is pointing outward $M_{*}$ through $A_{1}$ and inward $M_{*}$ through $A_{2}$.

Then, we glue back $A_{1}$ over $A_{2}$ by $\varphi$, i.e. we identify each point of $A_{1} \approx A$ with its image by $\varphi$ in $A_{2} \approx A$. We obtain a new manifold equipped with an oriented foliation. Since the topology of the new manifold only depends on the integer $n$, we will denote it by $M_{n}$. We call $n$-surgeries these operations of blowing up and glueing back by a diffeomorphism whose isotopy class corresponds to $n$. The proof of the following theorem can be easily extracted from [15]:

Theorem 2.1. For all positive integer $n$ there is a n-surgery leading to a foliation which is topologically conjugate to the foliation generated by an Anosov flow.

We will not reproduce here the proof of this theorem which is made in [15]. We will just indicate how the positivity condition of the integer $n$ appears in the proof.

Let $A_{0}^{\prime}$ be the annulus embedded in $M_{n}$ corresponding to $A_{1}$ and $A_{2}$. Let $x$ be an element of $A_{0}^{\prime}$ contained in a closed periodic leaf of $\Phi_{n}$. Clearly, if $\Phi_{n}$ is supposed to be the foliation generated by an Anosov flow, the first return map along $\Phi_{n}$ on the section $A_{0}^{\prime}$ has to be hyperbolic at the point $x$. We will show why it is true when $n$ is positive. For the sake of simplicity, we will assume that the periodic orbit through $x$ meets $A_{0}$ only at $x$.

First of all, we have to make a suitable choice of the glueing map $\varphi$. We define a parametrization $p: \mathbf{S}^{1} \times[0,2 \pi] \rightarrow A_{0}$ adapted to this purpose. We fix some parametrization $f: \mathbf{S}^{1} \rightarrow c_{2}$ preserving the orientations. Here, the orientation of $c_{2}$ is the induced one by by the orientation of $A_{0}$. Namely, if $\nu$ is a vector over $f(\theta)$ pointing inside $A_{0}$, then $\left(D_{\theta} f\left(\partial_{\theta}\right), \nu\right)$ must be a direct basis of $T_{f(\theta)} A$. Since $g_{0}^{s}$ and $g_{0}^{u}$ are transverse one to the other, and according to the orientations we have chosen, there is an open neighborhood $V$ of $c_{2}$ in $A_{0}$ and a positive real $\epsilon_{0}$ such that, for each pair $\theta<\theta^{\prime}$ of elements $2 \epsilon_{0}$-near of $\mathbf{R}$, the leaves $g_{0}^{u}\left(f\left(e^{i \theta}\right)\right)$ and $g_{0}^{s}\left(f\left(e^{i \theta^{\prime}}\right)\right)$ admit an unique point of intersection in $V$. Therefore, we can define the map $p: \mathbf{S}^{1} \times\left[0, \epsilon_{0}\right] \rightarrow V$ which associates to $\left(e^{i \theta}, \epsilon\right)$ the intersection in $V$ of $g_{0}^{s}\left(f\left(e^{i(\theta+\epsilon)}\right)\right)$ and $g_{0}^{u}\left(f\left(e^{i(\theta-\epsilon)}\right)\right)$. Rescaling the second factor, and replacing $A_{0}$ by the image of this map we obtain the desired parametrization of $A_{0}$. Remark that this map is $C^{1}$ since this is the class of differentiability of the foliations. The pull-back foliations $p^{*}\left(g_{0}^{u}\right)$ and $p^{*}\left(g_{0}^{s}\right)$ are generated by the vector fields $\lambda \partial_{\theta}+\partial_{y}$ and $-\lambda \partial_{\theta}+\partial_{y}$, respectively, where $\lambda$ is the scalar $\frac{\epsilon_{0}}{2 \pi}$. Let $f$ be an increasing map from 
$[0,2 \pi]$ onto $[0,1]$. Then, we define:

$$
\varphi\left(e^{i \theta}, y\right)=\left(e^{i(\theta+n f(y))}, y\right)
$$

Note that this map is as desired isotopically the Dehn twist of power $n$ relatively to the orientation we have chosen. So, the differential of $\varphi$ at the point $x$ is expressed in the basis $\left(\partial_{\theta}, \partial_{y}\right)$ by the matrix:

$$
\left(\begin{array}{cc}
1 & n f^{\prime}(y) \\
0 & 1
\end{array}\right)
$$

On the other hand, the first return map on $A$ along $\Phi^{t}$, where it is defined, preserves the foliations $g^{s}$ and $g^{u}$, contracting the first and dilating the second. Hence there are two real numbers $\mu^{u}$ and $\mu^{s}$ of absolute values greater than one such that the differential of this first return map multiplies $\lambda \partial_{\theta}+\partial_{y}$ and $-\lambda \partial_{\theta}+\partial_{y}$ by respectively $\mu^{u}$ and $\frac{1}{\mu^{s}}$. Note that since $M$ is oriented, the two eigenvalues $\mu^{u}$ and $\mu^{s}$ are of the same sign.

Since the first return map at $x$ along $\Phi_{n}$ is the composition of the two maps above, its differential at $x$ is given by the matrix:

$$
\left(\begin{array}{cc}
\frac{1}{2}\left(\mu^{u}+\frac{1}{\mu^{s}}\right) & \frac{n f^{\prime}(y)}{2}\left(\mu^{u}+\frac{1}{\mu^{s}}\right)+\frac{\lambda}{2}\left(\mu^{u}-\frac{1}{\mu^{s}}\right) \\
\frac{1}{2 \lambda}\left(\mu^{u}-\frac{1}{\mu^{s}}\right) & \frac{n f^{\prime}(y)}{2 \lambda}\left(\mu^{u}-\frac{1}{\mu^{s}}\right)+\frac{1}{2}\left(\mu^{u}+\frac{1}{\mu^{s}}\right)
\end{array}\right)
$$

Since $f^{\prime}(y)$ is never negative, this matrix is hyperbolic when $n$ is positive. It is not necessarily true when $n$ is negative with big absolute value.

Remark 2.2. In the construction above, since the annulus $A$ is transverse to the flow, it is transverse to the inverse $\Phi^{-t}$ too. At first glance, one might think that the positivity condition can be dropped out just by reversing the flow, because it corresponds to inverse the glueing map. But this is not correct, since when we reverse the flow, we exchange the foliations $g^{s}$ and $g^{u}$, and therefore we reverse the orientation on the annulus. Hence, if the glueing map is "negative" relatively to $\Phi^{t}$ its inverse is still "negative" relatively to the inverse of the flow.

Remark 2.3. The surgery above is traditionally used by taking a transverse annulus in the neighborhood of a periodic orbit of the flow. In this paper, we will use it in another situation: assume that we know the existence of a torus $T$ embedded in $M$ transversely to the Anosov flow. Then, $\mathcal{F}^{s}$ and 
$\mathcal{F}^{u}$ induces on $T$ two foliations that we denote by $g^{s}$ and $g^{u}$. We assume that these two foliations are without (bidimensional) Reeb components. Let $\mathcal{P}$ the set of primitive elements of $H_{1}(T)$, i.e. those which are represented by closed simple curves in $T$.

Lemma 2.4. Each element of $\mathcal{P}$ can be represented by a closed simple curve simultaneously transverse to $g^{s}$ and $g^{u}$.

Proof. Let $\gamma$ an element of $\mathcal{P}$. Assume first that $\gamma$ can be represented by some closed leaf $g_{0}$ of one of the two foliations, for example of $g^{s}$. Then, $g_{0}$ is transverse to $g^{u}$. Since the holonomy of $g^{s}$ along $g_{0}$ is hyperbolic, we can isotop slightly $g_{0}$ to some closed simple curve transverse to $g^{s}$, and which is still transverse to $g^{u}$. Thus, we are done in this case.

Hence we can assume that no closed leaf of $g^{s}$ or of $g^{u}$ represents $\gamma$. Since $g^{s}$ is without Reeb component, there is a closed simple curve $c$ transverse to $g^{s}$ whose homology class is $\gamma$. We can take such a curve $c$ in generic position with respect to $g^{u}$, i.e. satisfying the following three properties:

(1) the number of points of tangency between $c$ and $g^{u}$ is finite,

(2) near point such a point of tangency between $c$ and a leaf $g$ of $g^{u}$, the leaf $g$ remains locally on the same side of $c$,

(3) each leaf of $g^{u}$ contains at most one point of tangency with $c$.

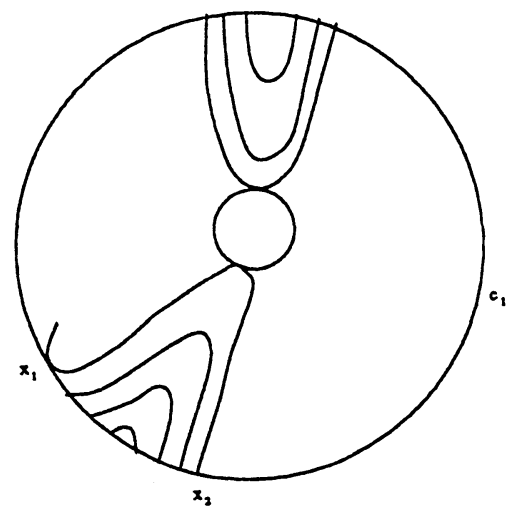

Figure 3: Surrounding a tangency. 


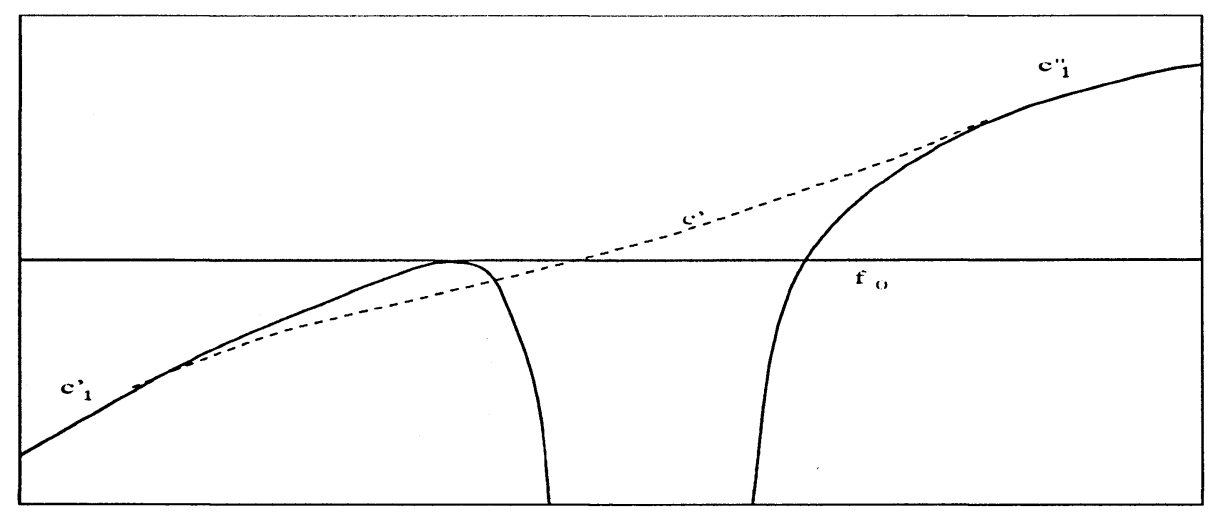

Figure 4: Trace of $c$ in $\mathcal{R}$.

Denote by $A$ the annulus obtained by cuting $T$ along $c$. The foliation $g^{s}$ induces on $A$ a foliation $f^{s}$ transverse to the boundary. Since no closed leaf of $g^{s}$ is homologous to $c$, the foliation $f^{s}$ has no periodic leaf. This implies that each leaf of $f^{s}$ is a path joining the two boundaries components of $\partial A$. In other words, they are the fibers of some fibration of $A$ over $\mathbf{S}^{1}$. The foliation $g^{u}$ induces another "foliation" $f^{u}$ which is tangent to $\partial A$ at a finite number of points. Consider one point of tangency of $c$ with $g^{u}$.

There is a boundary component $c_{1}$ of $\partial A$ which is tangent to $g^{u}$ at some point $x$, and an interval $I=] x_{1}, x_{2}$ [ in $c_{1}$ containing $x$ such that the holonomy along $f^{u}$ defines a map from $I$ onto $I$, fixing $x$ and exchanging the intervals ]$\left.x_{1}, x\right]$ and $\left[x, x_{2}[\right.$. The image by this application of each element $y \neq x$ of $I$ is the unique element $y^{\prime}$ of $I \backslash\{y\}$ belonging to the leaf $f_{y}^{u}$ of $f^{u}$ through $y$.

Take a maximal interval satisfying this property. Then the extremities $x_{1}$ and $x_{2}$ belong to the same leaf of $f^{u}$. There are only two possibilities (see figure 3 ):

(1) either this common leaf is tangent at some point to the other boundary component $c_{2}$ of $A$,

(2) either this common leaf is tangent at $c_{1}$ at $x_{1}$ or at $x_{2}$.

Note that according to the generic property (3), these two cases are disjoint, and in the second case the common leaf is transverse to $c_{1}$ at $x_{1}$ or $x_{2}$. 


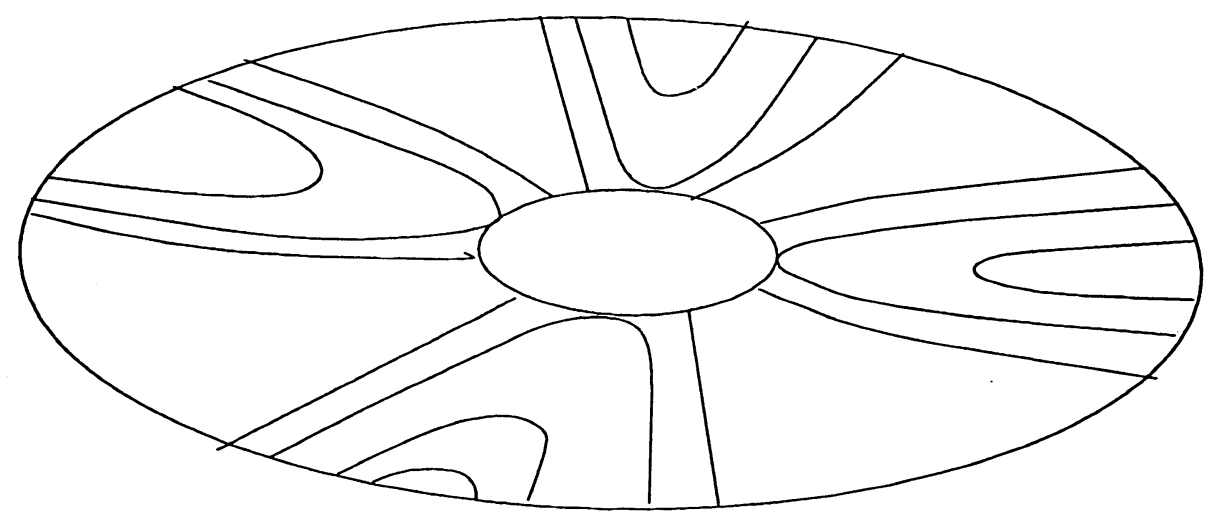

Figure 5: Reeb components of $f^{u}$.

We show now that isotoping $c$ we can delete one by one every occurence of the second case. Consider this case. We assume that the extremities where $c_{1}$ is tangent to $f^{u}$ is $x_{1}$. We denote by $f_{0}$ the $f^{u}$-leaf containing $x_{1}$ and $x_{2}$.

By pushing $f_{0} \subset T$ along the leaves of $f^{s}$ we obtain an embedding

$$
\mathcal{R}:[-1,1] \times[-1,1] \rightarrow T
$$

such that:

- it maps $\left[-\frac{1}{2}, \frac{1}{2}\right] \times\{0\}$ onto $f_{0}$,

- it maps each vertical $\{t\} \times[-1,1]$ into a leaf of $g^{s}$,

- it maps each horizontal $[-1,1] \times\{t\}$ into a leaf of $g^{u}$.

The preimage of $c$ by $\mathcal{R}$ is formed by two paths $c_{1}^{\prime}$ and $c_{1}^{\prime \prime}$ (see the figure 4).

Then, let $c^{\prime}$ be the graph of an increasing function from $[-1,1]$ into $[-1,1]$ which coincides with $c_{1}^{\prime} \cup c_{1}^{\prime \prime}$ near the vertical boundaries of $[-1,1] \times$ $[-1,1]$. The image $\mathcal{R}\left(c^{\prime}\right)$ meets $c$ along two intervals. Let $c^{\prime \prime}$ the connected component of the complement of these intervals in $c$ which doesn't contain $x$ : we check easily that $c^{\prime \prime} \cup \mathcal{R}\left(c^{\prime}\right)$ is a closed curve isotopic to $c$, transverse to $f^{s}$, and which has less points of tangency with $f^{u}$ than $c$.

Iterating this process we are led to situation where only the first case can occur, i.e. the case where every point of tangency between $f^{u}$ and $c_{1}$ is contained in a "Reeb component" of $f^{u}$ (see figure 5). 


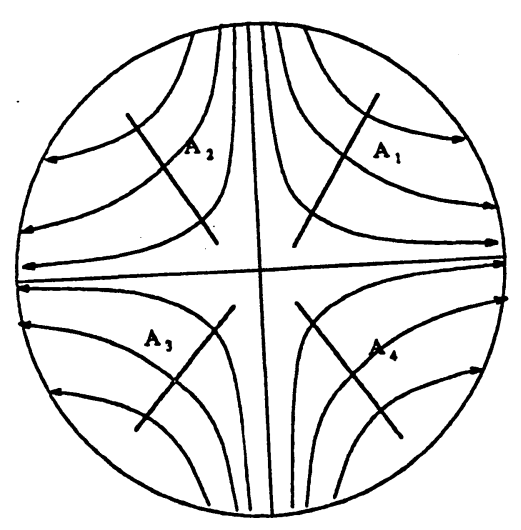

Figure 6: Transverse annuli in the neighborhood of a periodic orbit.

The composition of these "Reeb components" forms in $T$ Reeb components of $g^{u}$ - but we have excluded Reeb components in our hypothesis. Thus, the elimination of all the points of tangency of the first kind gives as wanted a representative of $\gamma$ transverse to both foliations.

According to the previous lemma, to each element $\gamma$ of $\mathcal{P}$ we can associate a closed transversal to both foliations. A suitable tubular neighborhood of this transverse curve will be an annulus satisfying the basket's property defined above, which enables to perform $n$-surgery. This surgery may be considered as follows: we cut $M$ along $T$, and we glue back the two toral boundary components by a Dehn twist along $\gamma$. Of course, the positivity condition prevents us from performing every Dehn twist, except if, as we will see in the following remark $2.5, \gamma$ can be representated by a closed leaf of $g^{s}$ or $g^{u}$.

We illustrate all this discussion in the context of suspensions. For every element of $G L(2, \mathbb{Z})$, let $\Phi_{A}$ be the suspension of the linear action of $A$ on the torus. This is a flow which is Anosov if and only if $A$ is hyperbolic, i.e. if and only if the absolute value of the trace of $A$ is greater than 2. Every global section of this flow is a torus on which we can perform the previous surgery (the traces of the weak foliations on this torus are without closed leaf: in particular, they have no Reeb's components). These surgeries don't affect the existence of global sections, therefore all the Anosov flows obtained 
by this way are topologically equivalent to suspensions $\Phi_{A^{\prime}}$ of elements of $G L(2, \mathbb{Z})$ (here we use Frank's theorem, cf. introduction). The positivity condition of Goodman's theorem translates in the following manner:

Fact. The traces of the matrix $A^{\prime}$ obtained by n-surgery from $A$ are of absolute values greater than the the absolute value of the trace of $A$.

Remark 2.5. We discuss here the classical presentation of the Goodman's surgery, which is usually related to the choice of some periodic orbit. Let $\theta_{0}$ be a periodic orbit of $\Phi^{t}$. There is some tubular neighborhood $W$ in which the flow is $C^{1}$-linearisable. In other words, there is a diffeomorphism $F_{0}$ between $W$ and $W_{B}$ realizing a topological equivalence between the restriction of $\Phi$ on $W$ and the restriction of $\Phi_{B}$ on $W_{B}$, where $B$ is a hyperbolic diagonal matrix, $\Phi_{B}$ the flow suspension of the action of $B$ on $\mathbf{R}^{2}, \theta_{B}$ the unique periodic orbit of $\Phi_{B}$, and $W_{B}$ a tubular neighborhood of $\theta_{B}$. The existence of such a linearization comes from the existence of the weak foliations. By the way, we have a good picture of the flow near $\theta_{0}$ (see figure 6).

We denote by $\mathcal{F}_{0}^{s}$ and $\mathcal{F}_{0}^{u}$ the traces on $W$ of the weak foliations. Note that since $M$ is oriented, the tubular neighborhood $W$ is always a solid torus. In $W$ we can find many transverse annuli satisfying the basket's property. Let's assume for a moment that we are in the case where both eigenvalues of $B$ are positive. Then, we can distinguish four types of transverse annuli, corresponding to the four "quadrants", i.e. the four connected components of the complement in $W$ of the two annuli in $W$ contained in the leaves of $\mathcal{F}_{0}^{s}$ and $\mathcal{F}_{0}^{u}$ through $\theta_{0}$. We choose representatives $A_{1}, A_{2}, A_{3}$ and $A_{4}$ of these four types. We choose the indexation such that $A_{1}$ and $A_{3}$ are in opposite quadrants. Then, $A_{2}$ and $A_{4}$ are in opposite quadrants too. Note that on $\partial W$ we have a particular oriented simple curve $l$ homotopic to $\theta_{0}$ in $W$ : one of the two connected components of the trace of the $\mathcal{F}_{0}^{s}$-leaf containig $\theta_{0}$. We fix some oriented meridian $m$ : it is a closed simple curve in $\partial W$ homotopically trivial in $W$. To each integer $n$, we thus can define the topological manifold $M_{n}$ obtained by performing the (usual) Dehn surgery, which identifies to points some curves in $\partial W$ homologous to $[m]+n[l]$. Now, we remark that every annulus $A_{i}$, since they satisfy the basket's property, is naturally oriented, and defines an orientation of $\partial W$. It is remarkable that the orientations defined by $A_{1}$ and $A_{3}$ are the same, and are opposite to the orientations defined by $A_{2}$ and $A_{4}$. Therefore, and up to a cyclic permutation of the indexation of the annuli, for each positive integer $n$, the ambient manifold of the Anosov flows obtained by $n$-Goodman-surgery 
along $A_{1}$ and $A_{3}$ is homeomorphic to $M_{n}$, and the ambient manifold of the flows obtained by $n$-Goodman-surgery along $A_{2}$ and $A_{4}$ is homeomorphic to $M_{-n}$. In other words, every manifold $M_{n}$, where $n$ is a positive or negative integer, admits an Anosov flow.

It may occur that the eigenvalues of $B$ are negative. In this case, the weak leaves through $\theta_{0}$ are Möbius bands. We have then only two quadrants, and therefore, only two types of transverse annuli. Moreover, the trace of the $\mathcal{F}_{0}^{s}$-leaf through $\theta_{0}$ is no more homotopic in $W$ to $\theta_{0}$, but to the double of $\theta_{0}$. We have to define the longitude $[l]$ as the half of the homology class defined by this trace. We define in this context $M_{n}$ as the manifold obtained by collapsing some fibers of a fibration on $\partial W$ where the homology class represented by the fibers is $2([m]+n[l])$. Performing $n$-Goodman-Dehn surgery, we obtain Anosov flows on $M_{n}$. If $n$ is positive, this surgery has to be performed along $A_{1}$, and if $n$ is negative, it has to be performed on the other annulus $A_{2}$.

In conclusion, the very pleasant feature of Goodman's surgery applied in the neighborhood of periodic orbits is that the positivity condition vanishes: it can be by-passed just by considering the good annulus.

Remark 2.6. Let $\Sigma$ a hyperbolic riemannian surface. This surface may be non-orientable. Recall that hyperbolic means that the metric is of constant curvature -1 . We denote by $\Phi_{0}^{t}$ the geodesic flow associated to $\Sigma$. We could start with some finite covering of this flow, but it is useless for our purpose. This flow is Anosov and its ambient manifold $M_{0}$ is the unitary tangent bundle of $\Sigma$. Consider a closed simple geodesic $c$ of $\Sigma$. We will assume here that $c$ is bicollared, i.e. that its tubular neighborhoods are not Möbius bands. A similar construction is possible when $c$ is not bicollared, but we will not need it for the present work. Let $T$ be the torus embedded in $M_{0}$ formed by the unitary vectors over $c$. It contains two periodic orbits $\theta_{1}$ and $\theta_{2}$ of $\Phi_{0}^{t}$, and outside these periodic orbits, it is transverse to the flow. The complement in $T$ of the periodic orbits is formed by two annuli $A_{1}^{0}$ and $A_{2}^{0}$. The weak foliations of $\Phi_{0}^{t}$ are transverse to the fibers of the fibration $M_{0} \rightarrow \Sigma$. Thus, they are suspensions of some actions of $\pi_{1}(\Sigma)$ on $\mathbf{S}^{1}$, which are projective actions. Hence the analog is true for the traces of the weak foliations on $T$. We deduce that every annular component $A_{j}^{0}$ of $T$ outside the periodic orbits satisfies the basket's property. Applying Goodman-Dehn surgery to these annuli we obtain the so-called examples of Handel-Thurston. These surgeries may be viewed as follows: there is an element $[l]$ of $H_{1}(T)$ well-defined up to the sign represented by any periodic orbit $\theta_{i}$. The surgery corresponds them to cutting the manifold along $T$, 
and glueing back by a Dehn twist along [l]: it is the presentation in [16].

The positivity condition of Goodman's theorem shows that only half of the Dehn twists on $T$ along $[l]$ are allowable. In fact, this operation is equivalent to a Goodman's surgery along a periodic orbit as described in the preceding remark, where the periodic orbit is one of the $\theta_{i}$. The two annular components $A_{1}^{0}$ and $A_{2}^{0}$ which are adjacent to $\theta_{i}$ in $T$ correspond, if we take the notations of remark 2.5, to two annuli $A_{i}$ and $A_{i+2}$ in opposite quadrants. Here is the explanation of the persistence of the positivity condition. But it can be by-passed, just by taking one of other two transverse annuli, let say, $A_{i+1}$. Isotoping $T$ we can assume that this annulus is contained in $T$. We recover in this way the missing Dehn twists along $[l]$. We obtain:

Proposition 2.7. Every manifold obtained from $M_{0}$ by cutting along $T$ and glueing back by a Dehn twist along [l] admits an Anosov flow.

One half of these Anosov flows, those which are obtained by choosing the annulus of surgery contained in $T$, are the examples of Handel-Thurston. We will extend this denomination to every Anosov flow obtained by DehnGoodman surgeries in the sense of remark 2.5 along periodic orbits over disjoint simple closed geodesics of $\Sigma$. All these Anosov flows are supported by graphmanifolds (remember that a graphmanifold is obtained by glueing seifert spaces along tori).

All of them are R-covered ([5], theorem B).

Remark 2.8. The $n$-surgeries preserves the property of preservation of a volume. In other words, any Goodman's surgery on an Anosov flow preserving a volume form leads to another Anosov flow which still preserves a volume form. It comes from the fact that every Dehn twist isotopy class admits representants preserving some volume on the annulus. As a corollary, the Handel-thurston's examples are volume preserving.

\section{Description of the BL-manifolds .}

Let $p: N \rightarrow \Sigma$ be the non trivial fibration by circles over twice punctured projective plane. Let $T_{1}$ and $T_{2}$ be the two boundary components of $\partial N$. A closed annulus embedded in $N$ is said vertical if its boundary is contained in $\partial N$ and is isotopic relatively to the boundary to the preimage by $p$ of a path in $\Sigma$. Such an annulus will be called essential if it is not $\partial$-parallel, i.e. if it is not isotopic to an annulus contained in $\partial N$. 
Proposition 3.1. The manifold $N$ satisfies all the following properties:

(1) $N$ is orientable,

(2) there are exactly two incompressible Klein bottles embedded in $N$ up to isotopy,

(3) every incompressible torus embedded in $N$ is isotopic to either $T_{1}$, or $T_{2}$, or to the boundary of a tubular neighborhood of an embedded Klein bottle,

(4) every essential vertical annulus whose boundary is contained in $T_{1}$ meets every essential vertical annulus whose boundary is contained in $T_{2}$.

Proof. Application of classical 3-dimensional theory. See for example [28].

We prescribe some orientation on $N$. The boundary is thus oriented. The BL-manifolds are the oriented manifolds obtained by glueing $T_{1}$ and $T_{2}$. Let $\sigma: \Sigma \rightarrow N$ be a section of $p$. We leave the proof of the following proposition to the reader:

Proposition 3.2. There are two involutions $J_{1}$ and $J_{2}$ from $N$ to itself such that:

- They both preserve the image of $\sigma$,

- $J_{1} H$ reverses the orientation of $N$ and preserves every fiber of $p$,

- $J_{2}$ is orientation preserving, permutes $T_{1}$ with $T_{2}$, and maps every fiber of $p$ on another fiber of $p$. Moreover, there is one and only one fiber of $p$ globally fixed by $J_{2}$. The restriction of $J_{2}$ to this fixed fiber is orientation reversing.

Let $T$ be the quotient $\mathbb{R}_{/ 2}^{2}$. Its first homology group is naturally identified with $\mathbb{Z}^{2}$. Let $[u]=(1,0)$ and $[v]=(0,1)$ be two generators of $H_{1}(T)$. We take the usual orientation on $\mathbf{R}^{2}$, and thus on $T$. Let $i_{1}: T \rightarrow T_{1}$ be an orientation preserving diffeomorphism, mapping the representatives of $[v]$ on loops homotopics to the fibers of $p$, and the representatives of $[u]$ on loops homotopics in $T_{1}$ to the boundary component contained in $T_{1}$ of the image of the section $\sigma$. Let $i_{2}$ be the composition of $i_{1}$ with the restriction 
of $J_{2}$ on $T_{1}$ : this is an orientation preserving diffeomorphism between $T$ and $T_{2}$. The action of $i_{2}$ on the homology groups is similar to this induced by $i_{1}$ : it maps $[v]$ on the homology class of the fibers (which is defined up to the sign), and $[u]$ to the homology class of the intersection of $T_{2}$ with the boundary of the image of $\sigma$. These parametrizations enable the definition of the glueing process: let $\Delta_{-}$be the set of the matrices with integer coefficients of determinant -1 . For every element $A$ of $\Delta_{-}$we define the manifold $M_{A}$, quotient of $N$ by the relation which identifies the points $i_{1}(y)$ and $i_{2}(x)$ of $T_{1}$ and $T_{2}$ if and only if the induced action of $A$ on $T$ maps $x$ on $y$. The BL-manifolds are exactly up to diffeomorphisms the manifolds of the form $M_{A}$. Note that $M_{A}$ is a circle bundle if and only if $A$ maps $(0,1)$ on $(0, \pm 1)$ i.e. if and only if $A$ is of the form:

$$
\left(\begin{array}{cc}
\epsilon & 0 \\
n & -\epsilon
\end{array}\right)
$$

where $\epsilon$ is \pm 1 . The projections of $T_{1}$ and $T_{2}$ in $M_{A}$ are equals. This common projection is an embedded torus that we denote by $T_{A}$, or $T$ when no confusion is possible.

Two different elements of $\Delta_{-}$may define the same BL-manifold. In the case of BL-manifolds which are circle bundles, we remark that all of them are fibering over a surface obtained by glueing the two boundary components of $\Sigma$. Such a surface is always diffeomorphic to the non-orientable surface of genus three $U_{3}$. Thus, the fibered BL-manifolds are characterized by their Euler class.

In a more general way, we can show that $M_{B}$ is always diffeomorphic to $M_{A}$ in the following cases:

- $B$ is the inverse of $A$ : the involution $J_{2}$ maps the relation between $T_{1}$ and $T_{2}$ defined by $A$ on the relation defined by $A^{-1}$,

- $B$ is conjugate to $A$ by the matrix:

$$
\left(\begin{array}{cc}
1 & 0 \\
0 & -1
\end{array}\right)
$$

The diffeomorphism between $M_{A}$ and $M_{B}$ is here the passage to the quotients of $J_{1}$,

- $B$ is of the form:

$$
B=\left(\begin{array}{cc}
1 & 0 \\
k & 1
\end{array}\right) A\left(\begin{array}{ll}
1 & 0 \\
k & 1
\end{array}\right)
$$


where $k$ is an integer. This diffeomorphism corresponds to a modification of the choice of the section $\sigma$. Indeed, when we modify the section, we modify $i_{1}$ and $J_{2}$, and thus $i_{2}$ as well. The new map $i_{1}^{k}$ still maps $[v]$ on fibers, but maps the homology class $[u]$ on $\left(i_{1}\right)_{*}\left([u][v]^{k}\right)$. The new map $i_{2}^{k}$ maps then $[u]$ on $\left(i_{1}\right)_{*}\left([u][v]^{-k}\right)$.

All these diffeomorphisms map $T_{A}$ onto $T_{B}$.

The converse is true: any diffeomorphism between two manifolds $M_{A}$ and $M_{B}$ is isotopic to a composition of diffeomorphisms of the form above: it is a corollary of the Waldhausen's classification of graphmanifolds (cf. [29]). We don't discuss further this part because it is useless for the present work.

In this paper we will use the following corollaries of these observations: the matrices $A^{+}$and $A^{-}$define the same BL-manifold, where:

$$
A^{+}=\left(\begin{array}{cc}
0 & 1 \\
1 & 0
\end{array}\right) \quad A^{-}=\left(\begin{array}{cc}
0 & -1 \\
-1 & 0
\end{array}\right)
$$

We conclude this section with the study of the fundamental group of BL-manifolds. We choose some base point $x_{0}$ of $N$ lying in the unique fiber fixed by $J_{2}$. We can further assume that $x_{0}$ lies in the image of the section $\sigma$ (thus, it's a fixed point of $\left.J_{2}\right)$. Let $Z$ be the fundamental group of $\left(N, x_{0}\right)$. We denote by $h$ the element of $Z$ represented by the fiber $p_{x_{0}}$ of $p$ through $x_{0}$. It is well defined up to the sign, but in our work, we will dispose of two vertical annuli embedded in $N$ : after isotopy, we can assume that one of these annuli contains $i_{1}((0,1))$ and $p_{x_{0}}$. Then, we can fix some orientation of $p_{x_{0}}$, and thus define $h$ without ambiguity, by imposing this oriented loop to be freely homotopic in the annulus to $i_{1}((0,1))$. We call it the fiber of $Z$. The group $Z$ admits the following presentation:

$$
Z=\left\langle a, b, c_{1}, c_{2}, h \mid a h a^{-1}=h^{-1}, b h b^{-1}=h^{-1}, c_{1}=a b, c_{2}=a^{-1} b\right\rangle
$$

Note that $h$ generates the pseudocenter of $Z . c_{1}$ and $c_{2}$ correspond to the boundary components of the image of $\sigma$. If we make a good choice of the isomorphism between $Z$ and the abstract group defined by the presentation, we can assume that the induced action of $J_{2}$ exchanges $c_{1}$ and $c_{2}$, and maps $h$ on $h^{-1}$.

Let $A$ be an element of $\Delta_{-}$.

$$
A=\left(\begin{array}{ll}
a & b \\
c & d
\end{array}\right)
$$


We note abusively $x_{0}$ the projection of $x_{0}$ in the quotient $M_{A}$. Let $\Gamma_{A}$ be the fundamental group of $\left(M_{A}, x_{0}\right)$. Sometimes, when no confusion is possible, we will denote it $\Gamma$. This group admits the following presentation:

$$
\begin{aligned}
& \Gamma_{A}=\left\langle a, b, c_{1}, c_{2}, h, t\right| a h a^{-1}=h^{-1}, b h b^{-1}=h^{-1}, \\
&\left.c_{1}=a b, c_{2}=a^{-1} b, t c_{2} t^{-1}=c_{1}^{a} h^{c}, t h t^{-1}=c_{1}^{-b} h^{-d}\right\rangle
\end{aligned}
$$

Once the isomorphism between $\Gamma_{A}$ and the group given by the presentation is chosen, a natural embedding of $Z$ in $\Gamma_{A}$ is defined. We will often assume that such an isomorphism is fixed and consider $Z$ as a subgroup of $\Gamma_{A}$. Furthermore, this identification being fixed, we will denote by $H$ the subgroup of $Z$ generated by $h$ and $c_{1}$. This subgroup is isomorphic to $\mathbb{Z}^{2}$, and is maximal for this property. In other words, it is the unique free abelian subgroup of $\Gamma$ containing $H$. Note also that $H$ is stable by division, i.e. an element of $\Gamma$ admitting a non trivial power in $H$ must belong to $H$.

\section{Handel-Thurston's examples on BL-manifolds.}

We describe in this section all the $\mathbf{R}$-covered Anosov flows on BLmanifolds, i.e. we prove the third assertion of theorem B.

Assume first that the BL-manifold $M$ is a circle bundle. According to $[13]^{4}$ any Anosov flow on $M$ is topologically equivalent to a finite covering of the geodesic flow of $U_{3}$. Hence the Euler class of the fibration of $M$ over $U_{3}$ must be the quotient of the Euler class of the unitary tangent bundle of $U_{3}$ by the index of this covering. But this last Euler class is -1! Thus, the covering must be trivial, i.e. every Anosov flow on $M$ is topologically equivalent to the geodesic flow of $U_{3}$.

This proves the first assertion of theorem B.

There is another family of $\mathbf{R}$-covered Anosov flows on BL-manifolds: the examples of Handel-Thurston (cf. remark 2.6). Note that the unique Handel-Thurston's examples supported by BL-manifolds are those obtained by Dehn-Goodman surgery near a periodic orbit of the geodesic flow of $U_{3}$ over a simple closed bicollared geodesic of $U_{3}$ (anyway, if it doesn't appear really obvious, this claim is a corollary of the result below). Note also that the choice of the closed geodesic is innocuous. Indeed, an hyperbolic metric on $U_{3}$ being fixed, let $\theta_{1}$ and $\theta_{2}$ be two different periodic orbits of the associated geodesic flow $\Phi_{0}^{t}$ over two closed simple bicollared loops $\gamma_{1}$ and

\footnotetext{
${ }^{4}$ The orientability hypothesis in [13] on the base surface is not necessary for the proof.
} 
$\gamma_{2}$ of $U_{3}$. Clearly, Goodman's $n$-surgeries preserve topological equivalences: assume that some topological equivalence between $\Phi_{0}^{t}$ and itself maps $\theta_{1}$ on $\theta_{2}$. Then, for every $n$-surgery near $\theta_{1}$, there is a $n$-surgery near $\theta_{2}$ leading to a flow topologically equivalent to the flow obtained by the first one. If the two loops $\gamma_{1}$ and $\gamma_{2}$ are equals, then we are done since in this case the application mapping each tangent vector to its inverse preserves the geodesic flow and maps $\theta_{1}$ on $\theta_{2}$. If not, the complements of these loops in $U_{3}$ are however both homeomorphic to the two punctured real projective plane. Therefore, there are two new hyperbolic metrics on $U_{3}$ and an isometry $F$ between these metrics mapping a closed geodesic $\gamma_{1}^{\prime}$ isotopic to $\gamma_{1}$ onto a closed geodesic $\gamma_{2}^{\prime}$ isotopic to $\gamma_{2}$. The geodesic flows associated to these metrics are both topologically equivalent to the initial flow $-1 \Phi_{0}^{t}$. One of these topological equivalences maps $\theta_{1}$ onto a periodic orbit over $\gamma_{1}^{\prime}$, and the other maps $\theta_{2}$ onto a periodic orbit over $\gamma_{2}^{\prime}$. Composing these topological equivalences with the differential of the isometry $F$, and modulo the observation above for the case of two periodic orbits over the same geodesic, we obtain a diffeomorphism preserving the foliation $\Phi_{0}$ and mapping $\theta_{1}$ on $\theta_{2}$.

Now, we show that conversely any $\mathbf{R}$-covered Anosov flow on a BLmanifold is topologically equivalent to a Handel-Thurston's example. Let $\left(M, \Phi^{t}\right)$ be such a flow. According to the discussion in the beginning of this section, we can assume that $M$ is not a circle bundle. Note that since $M$ is not a torus bundle over the circle, $\Phi^{t}$ is not topologically equivalent to a suspension.

We use the notations of section 3 . We denote by $h^{\prime}$ the element $t h t^{-1}$ of $\Gamma$. $h$ and $h^{\prime}$ belong both to the subgroup $H$ which correspond to the fundamental group of the torus $T$.

Assume that $h$ preserves some point in $Q^{s}$. Since $F i x(h)$ is $Z$-invariant, and since the isotropy group of any point of $Q^{s}$ for the action of $\Gamma$ is cyclic, $h$ admits an infinite number of fixed points. In particular, $h$ preserves the orientation of $Q^{s}$. The same argument shows that every element of $\Gamma$ commuting with $h$ (for example, belonging to $H$ ) preserves the orientation of $Q^{s}$. According to the preliminary section, there is an homeomorphism $\tau: Q^{s} \rightarrow Q^{s}$ commuting with the action of every element of $\Gamma$, if this action is orientation preserving. Furthemore, the set of attractive fixed points of $h$ is of the form $\tau^{k}(x)$, where $k$ describes all $\mathbb{Z}$. Hence, we can associate to each orientation preserving element $\gamma$ of $Z$ the unique integer $k(\gamma)$ such that:

$$
\gamma x=\tau^{k(\gamma)}(x)
$$

We denote by $Z_{0}$ the group of orientation preserving elements of $Z$. The map $k$ is a morphism between $Z_{0}$ and $\mathbb{Z}$. Its kernel is the isotropy group of 
$x$. Therefore, it is cyclic. Thus, $Z_{0}$ is an extension of $\mathbb{Z}$ by $\mathbb{Z}$. But it is an absurdity since this group clearly contains a free non-abelian group. Our preliminary assumption was wrong: $h$ acts freely on $Q^{s}$.

According to the lemma 7.2 of [4] some element $\gamma_{0}$ of $H$ fixes some point $x_{0}$ in $Q^{s}$. The generator of the isotropy group of this point has a non trivial power in $H$. According to the last remark of section 3 this generator must be in $H$. Hence, we can assume that $\gamma_{0}$ generates this isotropy group. In other words, the unique periodic orbit $\theta_{0}$ contained in the stable leaf corresponding to $x_{0}$ is freely homotopic to a closed simple loop contained in the torus $T$. In fact, according to the proposition 7.1 of [4] (see remark 7.14 of this paper too), modifying $T$ by some isotopy we can assume that it contains $\theta_{0}$. Since $\gamma_{0}$ is orientation preserving and commute with $h$, there is some integer $k$ such that, for every fixed point $x_{0}$ of $\gamma_{0}$ :

$$
h\left(x_{0}\right)=\tau^{k}\left(x_{0}\right)
$$

By the same way, since $h^{\prime}=t h t^{-1}$ belongs to $H$, there is an integer $l$ such that:

$$
h^{\prime}\left(x_{0}\right)=\tau^{l}\left(x_{0}\right)
$$

Since $t \circ \tau=\tau^{ \pm 1} \circ t$, we obtain:

$$
h^{\prime}\left(t^{-1} x_{0}\right)=\tau^{ \pm k}\left(t^{-1} x_{0}\right)
$$

Therefore, $l= \pm k$, and there is some integer $n$ such that:

$$
h^{\prime}=h^{ \pm 1} \gamma_{0}^{n}
$$

It means that, performing a $\pm n$-surgery on $\Phi^{t}$ near $\theta_{0}$, we are led to the case $h^{\prime}=h^{ \pm 1}$. Note that the ambient manifold of the new flow is still a BL-manifold because $\theta_{0}$ lies in $T$ : the surgery is a cut and paste on $M$ along $T$. The identity above means that this new manifold is a circle bundle. Hence, the new flow itself is topologically equivalent to the geodesic flow of $U_{3}$. Reversing the surgery, we see that $\Phi^{t}$ is an example of Handel-Thurston.

\section{A new family of Anosov flows.}

We consider here the BL-foliations and BL-flows as defined in the introduction. In [6] C. Bonatti and R. Langevin have shown:

Theorem. There is a BL-flow on $M_{A^{+}}$which is Anosov. 
$A^{+}$is the matrix defined in section 3. The diffeomorphism between $M_{A^{+}}$ and $M_{A^{-}}$induced by $J_{1}$ shows immediatly that the same is true for $M_{A^{-}}$. Moreover, this Anosov flow preserves some volume form.

The purpose of this section is to prove theorem A. Our method consists of showing that for every element $A$ of $\Delta_{-}$which is not a lower triangular matrix, there is a finite sequence of matrices $A_{1}, \ldots, A_{m}$ whose first term is $A^{+}$or $A^{-}$, and whose last term is $A$, and such that $M_{A_{i+1}}$ is obtained from $M_{A_{i+1}}$ by some Dehn-surgery supported in $T_{A_{i}}$. Futhermore, the surgery leading from $A_{1}$ to $A_{2}$ would have to satisfy the positivity condition of Goodman's theorem. Therefore, this first surgery gives some BL-foliation on $M_{A_{2}}$ which is Anosov. We impose thus inductively the similar positivity condition to the following surgeries. Hence, all the manifolds $M_{A_{i}}$ will support a BL-foliation which is Anosov: it will be true in particular for $M_{A}$. Note that this proof will show that all these Anosov BL-flows are obtained from the same BL-foliation on $N$, and that all of them are volume preserving (cf. remark 2.8).

After this description of the proof, we are left with the calculus.

We will always denote the coefficients of the element of $\Delta_{-}$in the following manner:

$$
A=\left(\begin{array}{ll}
a & b \\
c & d
\end{array}\right)
$$

Since we assume that $M_{A}$ is not a circle bundle, $b$ is not null. Assume that $M_{A}$ supports some Anosov BL-flow. Then, the annuli $A^{u}$ and $A^{s}$ embedded in $M_{A}$ corresponding to the vertical annuli in $N$ tangent to the BL-foliation which defines the BL-flow, are respectively contained in the unstable leaf and the stable leaf of the Anosov flow containing the periodic orbit $\theta_{0}$. Let $g^{s}$ and $g^{u}$ be the foliations induced by the weak foliations on the transverse torus $T_{A}$ : the closed leaves of $g^{s}$ are homotopic in $T_{A}$ to the boundary components of $A^{s}$. Therefore, their homology classes are $\pm[v]$. The closed leaves of $g^{u}$ are homotopic to the image by the glueing map of the boundary components of $A^{u}$ which are contained in $T_{2}$. Hence, their homology classes are $\pm(b[u]+d[v])$.

Note that every closed leaf of one of these foliations is a complete transversal for the other foliation, so none of them admits a Reeb component. Thus, according to the remark 2.3, for every prime pair of integers $(p, q)$, we can perform allowable Goodman's surgeries along the homology class $p[u]+q[v]$. Performing such a $n$-surgery along $p[u]+q[v]$ we deform $M_{A}$ to a new BL-manifold $M_{A^{\prime}}$ where $A^{\prime}$ is the composition to the left of $A$ 
by a Dehn twist matrix of the form:

$$
T_{n, p, q}=\left(\begin{array}{cc}
1-n p q & p^{2} n \\
-q^{2} n & 1+n p q
\end{array}\right)
$$

Our question is: given the integers $p$ and $q$, what is the sign of $n$ corresponding to the famous positivity condition?

As we observed in the remark 2.5 , this positivity condition vanishes when the surgery is performed near a periodic orbit. These particular surgeries in our context are exactly whose performed along closed leaves of $g^{s}$ or of $g^{u}$. In other words, there is no condition on $n$ when $p$ or $p d-q b$ is null.

We apply this observation to $A^{+}$and $A^{-}$: the compositions to the left of these two matrices by the matrices $T_{n, 1,0}$ are the matrices of the form:

$$
\left(\begin{array}{cc}
\epsilon n & \epsilon \\
\epsilon & 0
\end{array}\right)
$$

Hence:

Lemma 5.1. For every element of $\Delta_{-}$whose lower diagonal coefficient $d$ is null, the BL-manifold $M_{A}$ supports an Anosov BL-flow, obtained by Goodman's surgery from the Bonatti-Langevin's example.

Consider now the non trivial case, i.e. when $p(p d-q b)$ is not null. Lift $g^{s}$ and $g^{u}$ in the universal cover $\mathbf{R}^{2}$ of the torus. The leaves of the first lifted foliation are nearly "verticals", i.e. linear lines of null slope. The leaves of the second lifted foliation are nearly linear lines of slope $\frac{d}{b}$. Finally, the Dehn twist $T_{n, p, q}$ is along some curve which lifts to some line near the linear one of slope $\frac{p}{q}$. But the positivity condition of Goodman's theorem means exactly that $n$ can be positive if and only if the inverse of this last slope is not between the inverses of the slopes associated to the lifted foliations (see figure 7).

Hence:

Proposition 5.2. If $M_{A}$ supports an Anosov BL-flow, then the same is true for $M_{A^{\prime}}$ as soon as $A^{\prime}$ is of the form $T_{n, p, q} \circ A$ where $p=0$ or $n\left(\frac{d}{b}-\frac{q}{p}\right) \geq 0$.

We first deal with the case $d=-1$ :

Lemma 5.3. Let $A$ be an element of $\Delta_{-}$whose upper right coefficient $b$ is not null and whose lower diagonal coefficient d equals -1 . Then, $M_{A}$ supports an Anosov BL-flow obtained by Goodman's surgeries from the BonattiLangevin's example. 


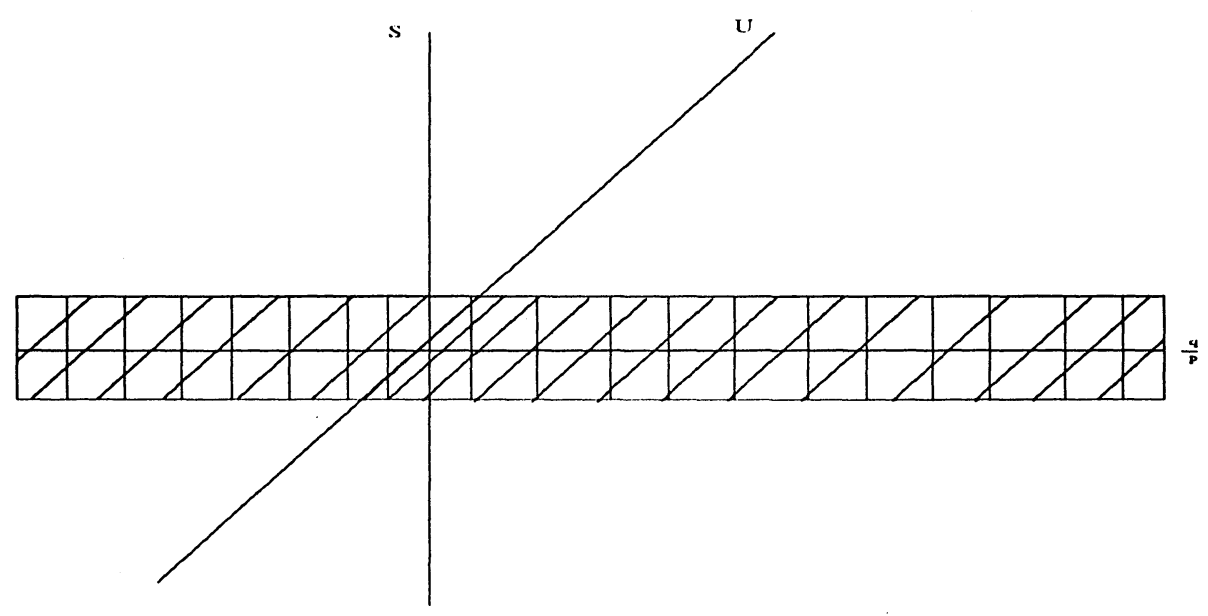

Figure 7: On the positivity condition.

Proof. The elements of $\Delta_{-}$satisfying the hypothesis of the lemma are the matrices $A(b, c) \quad(b \neq 0)$ where:

$$
A(b, c)=\left(\begin{array}{cc}
1-b c & b \\
c & -1
\end{array}\right)
$$

For every integer $c$ :

$$
\left(\begin{array}{ll}
1 & 0 \\
1 & 1
\end{array}\right) \circ\left(\begin{array}{cc}
1-c & 1 \\
c & -1
\end{array}\right) \circ\left(\begin{array}{ll}
1 & 0 \\
1 & 1
\end{array}\right)=\left(\begin{array}{cc}
2-c & 1 \\
1 & 0
\end{array}\right)
$$

Hence, according to lemma 5.1 and section 3 the BL-manifolds $M_{A(1, c)}$ support Anosov BL-flows.

For every integer $n$ we have:

$$
A(1-n, c)=T_{n, 1,0} \circ A(1, c)
$$

Applying the lemma 5.2, we obtain that all the manifolds $M_{A(b, c)}$ for $b>0$ support Anosov BL-flows.

Finally, since $A(-b,-c)$ is the conjugate of $A(b, c)$ by:

$$
\left(\begin{array}{cc}
1 & 0 \\
0 & -1
\end{array}\right)
$$


we obtain that $M_{A(b, c)}$ and $M_{A(-b,-c)}$ are diffeomorphic (see section 3). This proves the remaining case $b<0$.

The following proposition is exactly what we need to prove the theorem A:

Proposition 5.4. Let $A$ be an element of $\Delta_{-}$. If its coefficients $b$ and $d$ are not null and if $d \neq-1$, then there is a matrix $A^{\prime}$ - whose coefficients are $a^{\prime}, b^{\prime}, c^{\prime}$, and $d^{\prime}$ - and three integers $n, p$ and $q$ such that:

$$
\begin{aligned}
& -A^{\prime}=T_{p, q, n} \circ A \\
& -p=0 \text { or } n\left(\frac{d^{\prime}}{b^{\prime}}-\frac{q}{p}\right) \leq 0, \\
& -\left|d^{\prime}\right|<|d|, \\
& -b^{\prime} \neq 0 .
\end{aligned}
$$

Proof. We have the following equalities:

$$
\begin{aligned}
b^{\prime} & =(1-n p q) b+p^{2} n d \\
d^{\prime} & =-q^{2} n b+(1+n p q) d
\end{aligned}
$$

Note that $q=0$ implies $d^{\prime}=d$, so we will choose $q \neq 0$. We define:

$$
\alpha=n\left(\frac{b}{d}-\frac{p}{q}\right)
$$

The condition $\left|d^{\prime}\right|<|d|$ is equivalent to

$$
0>\left(d^{\prime}\right)^{2}-d^{2}=q^{4} d^{2} \alpha\left(\alpha-\frac{2}{q^{2}}\right) .
$$

Thus, it is equivalent to:

$$
0<\alpha<\frac{2}{q^{2}}
$$

Assuming $b^{\prime} \neq 0$ and $p \neq 0$ we define

$$
\Lambda=n\left(\frac{d^{\prime}}{b^{\prime}}-\frac{q}{p}\right) .
$$


An easy calculus shows:

$$
\Lambda=\frac{n\left(\frac{d}{b}-\frac{q}{p}\right)}{1+p^{2} n\left(\frac{d}{b}-\frac{q}{p}\right)}
$$

Thus, the sign of $\Lambda$ is the sign of

$$
p^{2}+\frac{1}{n\left(\frac{d}{b}-\frac{q}{p}\right)}=p^{2}-\frac{\frac{b}{d} \frac{p}{q}}{\alpha}
$$

Therefore, the two following systems are equivalents:

$$
\left\{\begin{array} { l } 
{ \Lambda \leq 0 \quad \text { or } \quad p = 0 } \\
{ b ^ { \prime } \neq 0 } \\
{ | d ^ { \prime } | < | d | } \\
{ q \neq 0 }
\end{array} \quad \left\{\begin{array}{l}
0<\alpha<\frac{2}{q^{2}} \\
p^{2} \alpha \leq \frac{b}{d} \frac{p}{q} \\
q \neq 0 \\
b \neq n p(q b-p d)
\end{array}\right.\right.
$$

The second inequality of the second system implies that $\frac{b}{d} \frac{p}{q}$ is not negative. Therefore the two systems are equivalent to:

$$
\left\{\begin{aligned}
\frac{b}{d} \frac{p}{q} & \geq 0 \\
n\left(\frac{b}{d}-\frac{p}{q}\right) & >0 \\
|n||b q-p d||q| & <2|d| \\
|n||b q-p d||p| & \leq|b| \\
b & \neq n p(q b-p d) \\
q & \neq 0
\end{aligned}\right.
$$

Proving the proposition 5.4 is equivalent to solve this system. Consider the euclidian division of $a$ by $b: a=-k b+r$ with $0 \leq r<|b|$.

We distinguish two cases. The first one is the case where $r$ is null. Then, $b$ divides $a$. Since $a d-b c=-1$, it implies $b= \pm 1$. Then, we can choose $p=0, q=1$ and $n=\frac{b d}{|d|}$. 
The second case we have to deal with is $r \neq 0$. In this case we choose $p=r$, and $q=c+k d$. With this choice $b q-p d=1: p$ and $q$ are relatively prime. Our choice of $n$ is the integer of absolute value 1 and with the sign of $\frac{b}{d}-\frac{p}{q}$. We have:

$$
q \neq 0
$$

It comes from the positivity of $p$, from the inequality $d \neq-1$ and from $b q-p d=1$.

$$
\begin{gathered}
n\left(\frac{b}{d}-\frac{p}{q}\right)>0 \\
|n||b q-p d||p|=r<|b| \\
n p(q b-p d)= \pm r \neq b
\end{gathered}
$$

$b q-p d=1$ implies $q=\frac{1+r d}{b}$. Hence:

$$
|n||b q-p d||q|=\frac{|1+r d|}{|b|} \leq \frac{1}{|b|}+\frac{r}{|b|}|d|
$$

It implies:

$$
|n||b q-p d||q|<2|d|
$$

Finally:

$$
\frac{b}{d} \frac{q}{p}=\frac{b}{d} \frac{1+r d}{b r}=\frac{1}{r}\left(\frac{1}{d}+r\right)
$$

Since $r$ and $|d|$ are both greater than one we obtain:

$$
\frac{b}{d} \frac{p}{q} \geq 0
$$

According (1), (2), (3), (4), (5) and (6) our choices satisfy the system $(S)$.

Let $A$ be any element of $\Delta_{-}$whose coefficient $b$ is not null. Applying inductively the proposition 5.4 we obtain some sequence $A^{1}, \ldots, A^{k}$, where $A^{1}=A$ and where the lower diagonal coefficient of the last term $A^{k}$ is equal to 0 or -1 . This sequence is finite since the absolute value of the lower diagonal coefficients is strictly decreasing. According to the lemmas 5.1 and 5.3, $M_{A^{k}}$ supports an Anosov BL-flow, and according to the proposition 5.2, 
$M_{A^{i}}$ supports an Anosov BL-flow as soon as $M_{A^{i+1}}$ does. This proves the theorem A.

\section{Topological transitivity of Anosov flows on BL-manifolds.}

To show the theorem B, we will have to prove that every Anosov flow on a BL-manifold is volume preserving. So, in particular:

Theorem 6.1. All Anosov flows on BL-manifolds are topologically transitive.

The proof of this theorem relies on [8] and on the following lemma:

Lemma 6.2. If some torus embedded in a BL-manifold is transverse to some Anosov flow, then it is isotopic to $T$.

Proof of theorem 6.1. The main theorem of [8] states that if $M$ support a non topologically transitive Anosov flow $\Phi^{t}$ then there is a finite family of disjoint tori embedded in $M$ transversely to $\Phi^{t}$ and such that every connected component of the complement in $M$ of the union of these tori contains one and only one basic set of $\Phi^{t}$. Moreover, the tori of this family are pairwise nonisotopic. In particular, the complement of this union should not be connected, since a non transitive Anosov flow admits at least two basic sets: an attractive one and a repulsive one.

According to lemma 6.2 , such a family of tori in a BL-manifold would be necessarily formed by a unique transverse torus isotopic to $T$. But $M$ is not disconnected by $T$ : contradiction.

Proof of lemma 6.2. Let $T^{\prime}$ be a torus embedded in the BL-manifold $M$ transverse to an Anosov flow $\Phi^{t}$. According to [9] or [8], $T^{\prime}$ is incompressible. Note also that, according to the section 4 , and since a $\mathbf{R}$-covered Anosov flow which is not a suspension cannot be transverse to a torus, the manifold $M$ is not a circle bundle. Hence $T^{\prime}$ is isotopic to a torus embedded in the characteristic manifold of $M$, which is $N \subset M$. According to the proposition 3.1, if $T^{\prime}$ is not isotopic to $T$, it is isotopic to the boundary of a tubular neighborhood $W$ of a Klein bottle $K$ embedded in $M$. Performing this isotopy on $\Phi^{t}$ we are led in this case to the situation where $\Phi^{t}$ is transverse to the boundary of $W$. In order to conclude, we will show that such a picture is impossible. 
Assume that we are in this case. There is some double covering of $W$ by $W^{\prime}=T^{2} \times[0,1]$. The restriction to $W$ of the flow and of the weak foliations lift in $W^{\prime}$. Reversing it if necessary, we can assume that the lifted local flow is inward $W^{\prime}$ through $\partial W^{\prime}$. Then we can reproduce the arguments of [8] or [4]: consider the lifting $G^{u}$ of the restriction of $\mathcal{F}^{u}$ to $W$. Let $A$ be an annular compact leaf of $G^{u}$ whose boundary is contained in the same boundary component of $W^{\prime}$. Then, all the positive semi-orbits of the local flow restricted to $A$ admits a $\omega$-limit point in $A$. But the restriction of an Anosov flow to an unstable leaf is "going to the infinity" for the induced topology: an orbit of such a restricted flow can admit a $\omega$-limit point if and only if it is periodic. Since no orbit meeting $\partial W$ is periodic we get a contradiction: such an annular leaf cannot exist. In particular, $G^{u}$ has no half-Reeb component. Since $\mathcal{F}^{u}$ has no compact leaf, $G^{u}$ is without Reeb component. Therefore, $G^{u}$ is a product foliation: it is conjugate to the product by $[0,1]$ of some foliation of $T^{2}$ (see for example [20]). Consider now any point of $\partial W^{\prime}$. Its positive orbit by the semiflow admits a $\omega$-limit point. Since for Anosov flows the periodic orbits are dense in the set of nonwandering points, it implies that the semi-flow admits some periodic orbit in $W^{\prime}$. Then, since $\Phi^{t}$ is incoming in $W$ through $T^{\prime}$, the $G^{u}$-leaf containing this periodic orbit cannot meet $\partial W^{\prime}$ : it is a contradiction with the description of $G^{u}$ we gave above.

\section{Existence of a transverse torus for a non-R-covered Anosov flow on a BL-manifold.}

During the following three sections, $\Phi^{t}$ is a non-R-covered Anosov flow on a BL-manifold $M$. According to section $4, M$ is not a circle bundle. To achieve the proof of theorem B, we have to show that $\Phi^{t}$ is a BL-flow, and, furthermore, that it is unique up to topological equivalence on $M$. In particular, such a flow will be necessarily topologically equivalent to the example on $M$ constructed in the section 5 .

We use all the notations of the preliminary section and of section 3 .

Lemma 7.1. The action of the fiber $h$ on $Q^{s}$ fixes some point.

Proof. Assume that $h^{2}$ acts freely on $Q^{s}$. Note that it preserves the orientation of $Q^{s}$. We distinguish two cases:

Case 1. Fix $\approx\left(h^{2}\right)$ is not empty: 
Near such a quasi-fixed point we can find some element $s$ comparable with its image by $h^{2}$. The union $I$ of the intervals $\left[h^{2 n}(s), h^{2 n+2}(s)\right]$ where $n$ describes $\mathbb{Z}$ is a open interval in $Q^{s}$. It is $h^{2}$-invariant. Since $h^{2}$ admits no fixed point, $I$ is the unique $h^{2}$-invariant interval: indeed, if $I^{\prime}$ is another $h^{2}$-invariant interval, then it must intersect $I$ (if not, the unique element of $\partial I$ disconnecting $I$ and $I^{\prime}$ would be fixed by $h^{2}$ ). This intersection should be connected since $Q^{s}$ is simply connected. Studying the dynamic of $h^{2}$ on $I$ and $I^{\prime}$, we obtain $I=I \cap I^{\prime}=I^{\prime}$.

But, for every element $\gamma$ of $Z, \gamma I$ is still a $h^{2}$-invariant interval. Hence $I$ is globally preserved by the action of whole $Z$. There is some element $t$ of $\Gamma$ such that the union of $t$ with $Z$ generates all $\Gamma$, and such that $h^{\prime}=t h t^{-1}$ belongs to $Z$. We can prove the same thing for $h^{\prime 2}$ as for $h^{2}$ : it preserves a unique open interval. But ${h^{\prime}}^{2}$ preserves both $I$ and $t I$. This proves that $I$ is preserved by $t$, and thus by every element of $\Gamma$. Since $\Phi^{t}$ is not $\mathbf{R}$-covered $I$ is not all $Q^{s}$. The boundary $\partial I$ is a closed $\Gamma$-invariant subset of $Q^{s}$, and, since it is formed by branching points, it is countable. In $M$, it corresponds to a closed subset saturated by $\mathcal{F}^{s}$ and transversely countable. Since $M$ is compact, this closed subset must admit a minimal closed invariant subset, and because of the countability property, this minimal subset should be a compact leaf of $\mathcal{F}^{s}$ : contradiction.

Case 2. The action of $h^{2}$ separates the points of $Q^{s}$ :

Then $h^{2}$ admits a fundamental axis (see preliminaries). This axis is $Z$-invariant. It is either a open interval or an union of intervals of the form $\left[s_{i}, s_{i+1}\right]$ where $s_{2 i} \approx s_{2 i-1}$. The first situation is excluded by the argument we used in the first case. In the second one, we obtain a map $\rho: Z_{0} \rightarrow \mathbb{Z}$ (here $Z_{0}$ is the set of the elements of $\Gamma$ commuting with $h$ ) defined by:

$$
\gamma s_{i}=s_{\rho(\gamma)+i}
$$

We derive a contradiction with the non-solvability of $Z_{0}$ using an argument applied in section 4 .

Therefore, $h^{2}$ admits fixed points in $Q^{s}$. Considering the action of $h$ on Fix $\left(h^{2}\right)$ we see that $h$ does admit a fixed point too (cf. e.g. [4]).

Lemma 7.2. The action of $h$ on $Q^{s}$ is orientation preserving.

Proof. If $h$ reverses the orientation, then its fixed point would be unique. This contradicts the fact that $Z$ preserves $F i x(h)$ and is not cyclic. 
The conjugate $h^{\prime}=t h t^{-1}$ is orientation preserving too, commutes with $h$, and admits a fixed point. We would be glad to apply theorem B of [4], but two hypothesis of this theorem is not fulfilled in our case. Namely:

- $\mathcal{F}^{s}$ is not transversely oriented,

- $M$ admits embeddings of the Klein bottle.

Anyway, we can use some of the arguments of the proof of this theorem:

Theorem 7.3. $T$ is isotopic to an embedded torus transverse to $\Phi^{t}$.

Proof. According to proposition 1.3, there is in $Q^{u}$ a fixed point $s$ of $h$ quasifixed by $h^{\prime}$ (the similar case where $h$ and $h^{\prime}$ are exchanged can be treated in the same manner). Let $y$ be the unique $h$-fixed point in $Q^{\Phi}$ contained in $s$ $\left(s\right.$ is considered as a leaf of $\left.\mathcal{G}^{s}\right)$. Then, $h^{\prime}(y)$ is fixed by $h$. According to the proposition 1.2 there is a sequence of lozenges joining $y$ and $h^{\prime}(y)$. Consider the union of the iterates of these lozenges by $h^{\prime}$. Since $s(y)$ and $s\left(h^{\prime} y\right)$ are not separated, we can easily show that this union forms a u-sequence of lozenges (see preliminaries). We denote by $\Omega$ the support of this u-sequence. It is an open subset of $Q^{\Phi}$ preserved by the actions of $h$ and $h^{\prime}$. We denote by $H^{\prime}$ the group generated by $h$ and $h^{\prime}$. Its action on $\Omega$ is proper and discontinuous.

Let $\widetilde{\Omega}$ be the preimage by $\pi^{\Phi}$ of $\Omega$. It is an open subset of $\widetilde{M}$ preserved by the action of $H^{\prime}$. Consider the quotient of this action: $\pi^{\Phi}$ induces a $\mathbf{R}$-fibration of this quotient over the quotient of $\Omega$ by the action of $H^{\prime}$. This last quotient is diffeomorphic to the torus. Hence, this fibration admits a section. This section lifts as a closed $H^{\prime}$-equivariant plane in $\widetilde{M}$ transverse to $\widetilde{\Phi}^{t}$. The projection by $\pi$ of this plane is an immersion in $M$ of the torus transverse to $\Phi^{t}$. Using cut and paste techniques (see for example [12]) we obtain an embedded transverse torus. According to the lemma 6.2 this transverse torus is isotopic to $T$.

\section{The flow is a BL-flow.}

According to the previous section, after a suitable isotopy, the torus $T$ is transverse to the flow. Cutting along $T$ we obtain an oriented foliation $\Phi_{1}$ on $N$ transverse to the boundary. We choose the identification between $M \backslash T$ and the interior of $N$ so that $\Phi_{1}$ is inward $N$ through $T_{1}$ and outward $N$ through $T_{2}$. Our goal is to prove that $\Phi_{1}$ is a BL-foliation. Since $h$ admits 
a fixed point in $Q^{s}$, there is a periodic orbit of $\Phi^{t}$ freely homotopic to the loops representing $h$. If this periodic orbit intersects $T$ then its intersection number with $T$ is non-trivial. This is impossible since $h$ belongs to $H$. Therefore, $\Phi_{1}$ admits closed periodic orbits. We denote by $\theta_{0}$ one of these periodic orbits (it might be unique, and we will see later that it is unique).

Since $\mathcal{F}^{s}$ and $\mathcal{F}^{u}$ are transverse to $T$ they induce two foliation $G^{s}$ and $G^{u}$ on $N$ transverse to the boundary. $\Phi_{1}$ is the foliation intersection of $G^{s}$ and $G^{u}$. We note $g_{i}^{s}$ and $g_{i}^{u}$ the traces of $G^{s}$ and $G^{u}$ on $T_{i} \quad(i=1,2)$. The argument used in the proof of lemma 6.2 shows that $G^{s}$ and $G^{u}$ are without half-Reeb components.

Let $\widetilde{N}$ be the universal cover of $N$ and $\widetilde{G}^{s}$ and $\widetilde{G}^{u}$ the liftings in $\widetilde{N}$ of $G^{s}$ and $G^{u}$. The interior of the leaves of this foliations are homeomorphic to the plane. Hence the leaf spaces $R^{s}$ and $R^{u}$ of $\widetilde{G}^{s}$ and $\widetilde{G}^{u}$ are connected simply connected one manifolds. The group $Z$ acts naturally on these leaf spaces. Let $F i x^{s}$ and $F i x^{u}$ be the sets of $h$-fixed points in $R^{s}$ and $R^{u}$. They are non empty since they contain the liftings of the leaves of $G^{s}$ and $G^{u}$ through $\theta_{0}$.

Lemma 8.1. Each foliation $G^{s}$ and $G^{u}$ admits a compact leaf which is a vertical annulus.

Proof. Fix ${ }^{s}$ and Fix ${ }^{u}$ are both countable, and their boundaries are countable too. Therefore their closures are closed countable $Z$-invariant subsets of $R^{s}$ and $R^{u}$. This implies that $G^{s}$ and $G^{u}$ admit both closed leaves. These compact leaves are contained in leaves of $\mathcal{F}^{s}$ or $\mathcal{F}^{u}$. Since $T_{1}$ and $T_{2}$ are incompressible and since no leaf of $g_{i}^{s}$ or $g_{i}^{u}$ is homotopically trivial in $T_{i}$, the boundaries of these compact leaves of $G^{s}$ and $G^{u}$ are homotopically non trivial in the leaves of $\mathcal{F}^{s}$ or $\mathcal{F}^{u}$ containing them. Therefore, they are homeomorphic to the annulus or to the Möbius band. Compact incompressible surfaces embedded in circle bundles are classified (see [28]). In our case, every compact leaf is either a $\partial$-parallel annulus or a vertical annulus. The first case implies the existence of half-Reeb components, which is impossible. Therefore, the closed leaves are vertical annuli.

Lemma 8.2. The foliation $G^{s}$ (respectively $G^{u}$ ) admits a unique closed leaf $A^{s}$ (resp. $\left.A^{u}\right)$. The boundary of $A^{s}$ is contained in $T_{1}$, and the boundary of $A^{u}$ is contained in $T_{2}$. The intersection of these two vertical annuli is a periodic orbit of $\Phi_{1}$. 
Proof. Every compact leaf of $G^{s}$ and $G^{u}$ induces compact leaves of $g_{i}^{s}$ and $g_{i}^{u}$. These loops are freely homotopics in $N$ to the fibers of $p: N \rightarrow \Sigma$. The glueing map $T_{2} \rightarrow T_{1}$ maps closed leaves of $g_{2}^{s}$ onto closed leaves of $g_{1}^{s}$. If some compact leaf of $G^{s}$ is joining $T_{1}$ and $T_{2}$, it would map the fibers of $p$ in $T_{2}$ on loops homotopic in $T_{1}$ to the fibers of $p$. This is impossible since $M$ is not a circle bundle. Therefore, the boundaries of a closed leaf are contained in the same boundary component of $N$. Since $\Phi_{1}$ is inward through $T_{1}$ and outward through $T_{2}$ the boundary of a closed leaf of $G^{s}$ is contained in $T_{1}$, and the boundary of a closed leaf of $G^{u}$ is contained in $T_{2}$. According to the fourth assertion of proposition 3.1 every closed leaf of $G^{s}$ intersects every closed leaf of $G^{u}$. Such an intersection is necessarily formed by periodic orbits of the flow. Since every weak leaf admits at most one periodic orbit, $G^{s}$ admits a unique closed leaf, $G^{u}$ admits a unique closed leaf, and the intersection between these closed leaves is a single periodic orbit.

Lemma 8.3. $A^{s}$ (respectively $A^{u}$ ) is the unique leaf of $G^{s}$ (resp. $G^{u}$ ) containing a loop freely homotopic in $N$ to the fibers of $p$.

Proof. The proof of lemma 8.1 shows that the lemma 8.2 can be translated in the following way: let $F_{0}^{s}$ be the subset of $R^{s}$ formed by the liftings of the closed leaf $A^{s}$ of $G^{s} . F_{0}^{s}$ is the unique minimal $Z$-invariant closed subset of $R^{s}$. Fix $x^{s}$ is a dicrete $Z$-invariant subset containing $F_{0}^{s}$. According to the previous observation $F i x^{s} \backslash F_{0}^{s}$ if not empty must contain $F_{0}^{s}$ in its closure! The only possibility is the equality $F_{0}^{s}=F i x^{s}$. The proof of the analog property for $G^{u}$ is the same.

The previous lemma admits the following corollary:

Corollary 8.4. The foliation $\Phi_{1}$ admits a unique closed periodic leaf. The two boundary components of $A^{s}$ are the unique closed leaves of $g_{1}^{s}$, and two boundary components of $A^{u}$ are the unique closed leaves of $g_{2}^{u}$.

Proposition 8.5. The foliation $\Phi_{1}$ is a BL-foliation.

Proof. The only property which remains to prove is that every leaf of $T_{1}$ which is not contained in $A^{s}$ or $A^{u}$ is joining $T_{1}$ and $T_{2}$. Assume it is not the case. Then, according to lemma 8.4 such a leaf is not periodic. It must admit a limit point in $N$. This limit point cannot be the periodic orbit $\theta_{0}$, since every orbit passing near $A^{s} \cup A^{u}$ which is not contained in $A^{s} \cup A^{u}$ 
meets both $T_{1}$ and $T_{2}$. The orbit of this limit point lies entirely outside $A^{s} \cup A^{u}$. The shadow lemma ([7]) implies that arbitrarly near this limit orbit $\Phi_{1}$ admits a periodic orbit. Contradiction.

\section{Coding the orbits of Anosov BL-flows.}

According to the corollary 8.4 of the previous section:

Lemma 9.1. The foliation $g^{s}$ (respectively $g^{u}$ ) induced by $\mathcal{F}^{s}$ (respectively $\left.\mathcal{F}^{u}\right)$ on $T$ is without Reeb component and admits exactly two closed leaves.

Let $\widetilde{T}^{0}$ be the unique lifting of $T$ in $\widetilde{M}$ preserved by the subgroup $H$ of $\Gamma$. It disconnects $\widetilde{M}$ and is transverse to $\widetilde{\Phi}$. Therefore, the restriction of $\pi^{\phi}$ to $\widetilde{T}^{0}$ is an homeomorphism on its image. We denote by $\Omega$ this image. The following lemma is an easy corollary of lemma 9.1 :

Lemma 9.2. $\Omega$ is the support of a s-sequence of lozenges $\left(\mathcal{L}_{n}\right)_{n \in}$ and of a $u$-sequence of lozenges $\left(\mathcal{L}_{n}^{\prime}\right)_{n \in}$.

We denote by $\left(x_{i}\right)_{i \in}$ the vertices of $\left(\mathcal{L}_{n}\right)_{n \in}$ and $\left(y_{i}\right)_{i \in}$ the vertices of $\left(\mathcal{L}_{n}^{\prime}\right)_{n \in}$.

Let $\mathcal{U}$ be the preimage $\pi^{-1}(T)$. Let $\mathcal{T}$ be the set of the connected components of $\mathcal{U}$. In other words, it is the set of the $\Gamma$-iterates of $\widetilde{T}^{0}$. Let $W_{1}$ and $W_{2}$ be the closures in $M$ of the two connected components of $M \backslash\left\{T \cup A^{s} \cup A^{u}\right)$. We define on $\mathcal{T}$ the following preorder: we denote by $\widetilde{T} \vdash_{1} \widetilde{T}^{\prime}$ if there is some lifting $\widetilde{W}$ of $W_{1}$ or $W_{2}$ in $\widetilde{M}$ such that:

(1) $\partial \widetilde{W}$ meets $\widetilde{T}$ and $\widetilde{T}^{\prime}$,

(2) $\widetilde{\Phi}^{t}$ is inward $\widetilde{W}$ through $\widetilde{T} \cap \widetilde{W}$ and outward $\widetilde{W}$ through $\widetilde{T} \cap \widetilde{W}$.

Since $\Phi^{t}$ is a BL-flow it is equivalent to require that there is an element of $\widetilde{T}$ whose positive orbit meets $\mathcal{U}$, and that the first intersection of this positive orbit with $\mathcal{U}$ lies on $\widetilde{T}^{\prime}$. The advantage of the definition above is that it does not depend on the flow, but only on $\left(M, T, A^{s}, A^{u}\right)$. We complete inductively $\vdash_{1}$ as an order $\vdash$ on $\mathcal{T}$ forcing the transitivity: we denote by $\widetilde{T} \vdash \widetilde{T}^{\prime}$ if there is a sequence $\left(\widetilde{T}_{1}, \ldots, \widetilde{T}_{n}\right)$ of elements of $\mathcal{T}$ such that:

$$
\widetilde{T}=\widetilde{T}_{1} \vdash_{1} \widetilde{T}_{2} \vdash_{1} \ldots \vdash_{1} \widetilde{T}_{n}=\widetilde{T}^{\prime}
$$


Note that if $\widetilde{T} \vdash \widetilde{T}^{\prime}$ the sequence $\left(\widetilde{T}_{1}, \ldots, \widetilde{T}_{n}\right)$ satisfying $(*)$ is unique, and is a subsequence of every 1 -increasing sequence starting from $\widetilde{T}$ and ending with $\widetilde{T}^{\prime}$.

We can give another definition of the order $\vdash$ :

Lemma 9.3. Let $\widetilde{T}=\gamma \widetilde{T}^{0}$ and $\widetilde{T}^{\prime}=\gamma^{\prime} \widetilde{T}^{0}$ be two elements of $\mathcal{T}$. The inequality $\widetilde{T} \vdash \widetilde{T}^{\prime}$ holds if and only if the intersection

$$
\pi^{\Phi}(\widetilde{T}) \cap \pi^{\Phi}\left(\widetilde{T}^{\prime}\right)=\gamma \Omega \cap \gamma^{\prime} \Omega
$$

is not empty and is contained in one of the lozenge $\gamma \mathcal{L}_{n}$. Moreover, if $\widetilde{T}-\widetilde{T}^{\prime}$ the intersection $\gamma \Omega \cap \gamma^{\prime} \Omega$ is saturated by the restriction of $\mathcal{G}^{s}$ to $\gamma \Omega$.

Proof. All the proof is based on the following obvious fact: let $\Omega$ and $\Omega^{\prime}$ be the supports of two s-sequences of lozenges. Then, either $\Omega$ and $\Omega^{\prime}$ are equals, either they are disjoint, either their intersection lies entirely in a lozenge of one of them.

Assume that $\gamma \Omega \cap \gamma^{\prime} \Omega$ is not empty and is contained in one of the lozenges $\gamma \mathcal{L}_{n}$. Then, there is some orbit of $\widetilde{\Phi}^{t}$ meeting $\gamma \widetilde{T}^{0}$ and $\gamma^{\prime} \widetilde{T}^{0}$. Furthermore, the fact that the intersection lies in some $\gamma \mathcal{L}_{n}$ shows that this orbit meets $\gamma \widetilde{T}^{0}$ before $\gamma^{\prime} \widetilde{T}^{0}$. Considering the elements of $\mathcal{T}$ that this orbit intersects, we obtain a sequence of elements of $\mathcal{T}$ satisfying $(*)$.

Inversely: assume that $\gamma \widetilde{T}^{0} \vdash_{1} \gamma^{\prime} \widetilde{T}^{0}$. Then, $\gamma \Omega \cap \gamma^{\prime} \Omega$ is not empty. Since $\gamma \widetilde{T}^{0}$ and $\gamma^{\prime} \widetilde{T}^{0}$ are different, their projections $\gamma \Omega$ and $\gamma^{\prime} \Omega$ are different. Therefore, the intersection $\gamma \Omega \cap \gamma^{\prime} \Omega$ is contained either in some $\gamma \mathcal{L}_{n}$, either in some $\gamma^{\prime} \mathcal{L}_{n}$. Since the orbits of $\widetilde{\Phi}^{t}$ intersecting both $\gamma \widetilde{T}^{0}$ and $\gamma^{\prime} \widetilde{T}^{0}$ meet first $\gamma \widetilde{T}^{0}$, we are in the first case. In other words, there is an integer $n$ such that:

$$
\gamma \Omega \cap \gamma^{\prime} \Omega \subset \gamma \mathcal{L}_{n}
$$

Moreover, this intersection is saturated by the restriction of $\mathcal{G}^{s}$ to $\gamma \mathcal{L}_{n}$. Assume now that we have $\gamma^{\prime} \widetilde{T}^{0} \vdash_{1} \gamma^{\prime \prime} \widetilde{T}^{0}$. Then, for some integer $n^{\prime}$ we have:

$$
\gamma^{\prime} \Omega \cap \gamma^{\prime \prime} \Omega \subset \gamma^{\prime} \mathcal{L}_{n^{\prime}}
$$

Since every leaf of the restriction of $\mathcal{G}^{s}$ to $\gamma^{\prime} \Omega$ intersects $\gamma \mathcal{L}_{n}$ we see that $\gamma \Omega \cap \gamma^{\prime \prime} \Omega$ is not empty. Moreover, this intersection is contained in $\gamma \mathcal{L}_{n}$. The proof is completed by an easy induction.

Let $\overline{\mathcal{D}}^{+}$be the set of the infinite sequences $\left(\widetilde{T}_{0}, \ldots, \widetilde{T}_{i}, \ldots\right)$ such that for every non negative integer $i$ we have $\widetilde{T}_{i} \vdash_{1} \widetilde{T}_{i+1}$. Let $\mathcal{D}^{+}$be the set of the 
elements of $\mathcal{U}$ whose positive orbit meets an infinite number of elements of $\mathcal{T}$. To each element $x$ of $\mathcal{D}^{+}$we associate its itinerary, i.e. the sequence of elements of $\mathcal{T}$ that its positive orbit meets successively. It defines a map $I^{+}: \mathcal{D}^{+} \rightarrow \overline{\mathcal{D}}^{+}$.

Lemma 9.4. The map $I^{+}$is surjective.

Proof. Let $\left(\widetilde{T}_{i}\right)_{i \in \mathbf{N}}$ be an element of $\overline{\mathcal{D}}^{+}$. According to the lemma 9.3 there is an integer $n$ such that for every index $i$, the intersection between $\pi^{\Phi}\left(\widetilde{T}_{0}\right)$ and $\pi^{\Phi}\left(\widetilde{T}_{i}\right)$ is not empty and is contained in $\gamma \mathcal{L}_{n}$ (here $\gamma$ is an element of $\Gamma$ sending $\widetilde{T}^{0}$ on $\widetilde{T}_{0}$ ). Moreover, all these intersections are saturated by the restriction of $\mathcal{G}^{s}$ to $\gamma \Omega$. Let $I^{s}$ be the leaf space of this restricted foliation on $\gamma \Omega . I^{s}$ is homeomorphic to the real line, and the intersections $\pi^{\Phi}\left(\widetilde{T}_{0}\right) \cap$ $\pi^{\Phi}\left(\widetilde{T}_{i}\right)$ correspond to a strictly decreasing sequence of subintervals. The intersection $I_{+\infty}$ of this decreasing subintervals is therefore non empty. Let $x$ be an element of $\widetilde{T}_{0}$ whose image by $\pi^{\Phi}$ lies on a leaf in $\gamma \mathcal{L}_{n}$ corresponding to an element of $I_{+\infty}$ : the itinerary of $x$ is exactly the sequence $\left(\widetilde{T}_{i}\right)_{i \in \mathrm{N}}$.

Lemma 9.5. Two elements of $\mathcal{D}^{+}$have the same itinerary if and only if they belongs to the same leaf of the trace on $\mathcal{U}$ of $\widetilde{\mathcal{F}}^{s}$.

Proof. We denote by $g_{\mathcal{U}}^{s}$ the trace of $\widetilde{\mathcal{F}}^{s}$ on $\mathcal{U}$. It appears implicitly is the proof of the previous lemma that $I^{+}$is $g_{\mathcal{U}}^{s}$-invariant. The other implication is equivalent to the following assertion: the interval $I_{+\infty}$ defined in the proof of lemma 9.4 contains one and only one point. Assume it is not the case: then, this interval would be of non empty interior. Therefore, the interior of the set of the elements of $\mathcal{D}^{+}$whose itinerary corresponds to $I_{+\infty}$ is not empty. Since $\Phi^{t}$ is topologically transitive (theorem 6.1) it contains an element of $\widetilde{M}$ over an element of the leaf $\mathcal{F}^{s}\left(\theta_{0}\right)$. This is impossible, since the positive orbit of an element of $\mathcal{F}^{s}\left(\theta_{0}\right)$ cannot admit an infinite number of intersection with the torus $T$.

Considering the negative orbits of the elements of $\mathcal{U}$ we construct a map $I_{\infty}: \mathcal{D}_{\infty} \rightarrow \overline{\mathcal{D}}_{\infty}$ such that:

- $\mathcal{D}_{\infty}$ is the the set of elements of $\mathcal{U}$ whose positive and negative orbits by $\widetilde{\Phi}^{t}$ meet both an infinite number of elements of $\mathcal{T}$,

- $\overline{\mathcal{D}}_{\infty}$ is the set of infinite sequences $\left(\widetilde{T}_{i}\right)_{i \in}$ such that for every integer $i$ we have $\widetilde{T}_{i} \vdash{ }_{1} \widetilde{T}_{i+1}$, 
- for every element $x$ of $\mathcal{D}_{\infty} I_{\infty}(x)$ is the sequence of the elements of $\mathcal{T}$ successively met by the $\widetilde{\Phi}^{t}$-orbit of $x$.

According to the lemmas 9.4 and 9.5 , and since every leaf of $g_{\mathcal{U}}^{s}$ intersects every leaf of $g_{\mathcal{U}}^{u}$ on at most one point we have:

Proposition 9.6. The map $I_{\infty}$ is objective.

Let $\mathcal{P}: \mathcal{D}_{\infty} \rightarrow \mathcal{D}_{\infty}$ be the first return map on $\mathcal{U}$. Let $\sigma: \overline{\mathcal{D}}_{\infty} \rightarrow \overline{\mathcal{D}}_{\infty}$ be the shift map. We have the equality:

$$
I_{\infty} \circ \mathcal{P}=\sigma \circ I_{\infty}
$$

Let $\mathcal{D}_{\infty}^{\Phi}$ be the image of $\mathcal{D}_{\infty}$ by $\pi^{\Phi}$. It is a dense subset of $Q^{\Phi}$ with empty interior. It can be identified with the quotient of $\mathcal{D}_{\infty}$ by the action of $\mathcal{P}$. Let $\widehat{\mathcal{D}}_{\infty}$ be the quotient of $\overline{\mathcal{D}}_{\infty}$ by the action of the shift map $\sigma$. We have a objection:

$$
\bar{I}_{\infty}: \mathcal{D}_{\infty}^{\Phi} \rightarrow \widehat{\mathcal{D}}_{\infty}
$$

The action of $\Gamma$ on $\mathcal{T}$ induces a $\sigma$-equivariant action on $\overline{\mathcal{D}}_{\infty}$, hence an action on $\widehat{\mathcal{D}}_{\infty}$. On the other hand, $\mathcal{D}_{\infty}^{\Phi}$ is $\Gamma$-invariant. Obviously:

Lemma 9.7. The map $\bar{I}_{\infty}$ commutes with the actions of $\Gamma$ on $\mathcal{D}_{\infty}^{\Phi}$ and $\widehat{\mathcal{D}}_{\infty}$.

Finally, we have other structures on $\Gamma$ on $\mathcal{D}_{\infty}^{\Phi}$ and $\overline{\mathcal{D}}_{\infty}$ preserved by $\bar{I}_{\infty}$ : Let $\mathcal{R}^{s}$ be the relation on $\widehat{\mathcal{D}}_{\infty}$ identifying two elements if and only if they admit representatives $\left(\widetilde{T}_{i}\right)_{i \in}$ and $\left(\widetilde{T}_{i}^{\prime}\right)_{i \in}$ such that for all positive integers $i$ the planes $\widetilde{T}_{i}$ and $\widetilde{T}_{i}^{\prime}$ are equals. In a similar way, we define a relation identifying two elements of $\widehat{\mathcal{D}}_{\infty}$ "sharing the same negative part". According to the lemma 9.5:

Lemma 9.8. $\bar{I}_{\infty}$ maps the relation "to be on the same leaf of $\mathcal{G}^{s}$ " (respectively of $\mathcal{G}^{u}$ ) on the relation $\mathcal{R}^{s}$ (respectively $\mathcal{G}^{u}$ ).

Consider the quotient $K^{s}=\mathcal{D}_{\infty / \mathcal{G}^{s}}^{\Phi}$. It can be considered as a subset of $Q^{s}$. Therefore, an orientation of $Q^{s}$ induces an order $\prec^{s}$ on $K^{s}$.

Let us fix an transverse orientation of $A^{s} \subset N$. Since $A^{s}$ is contained in a leaf of $Q^{s}$, this defines an orientation of $Q^{s}$, hence fixes the choice of the order $\prec s$. With the orientation of $T$ induced by the orientations of $M$ and $\Phi$, this transverse orientation defines an order between the connected components of the preimage of $A^{s}$ meeting $\widetilde{T}^{0}$ and contained in the "positive" part of $\widetilde{M} \backslash \widetilde{T}^{0}$ (see figure 8). 


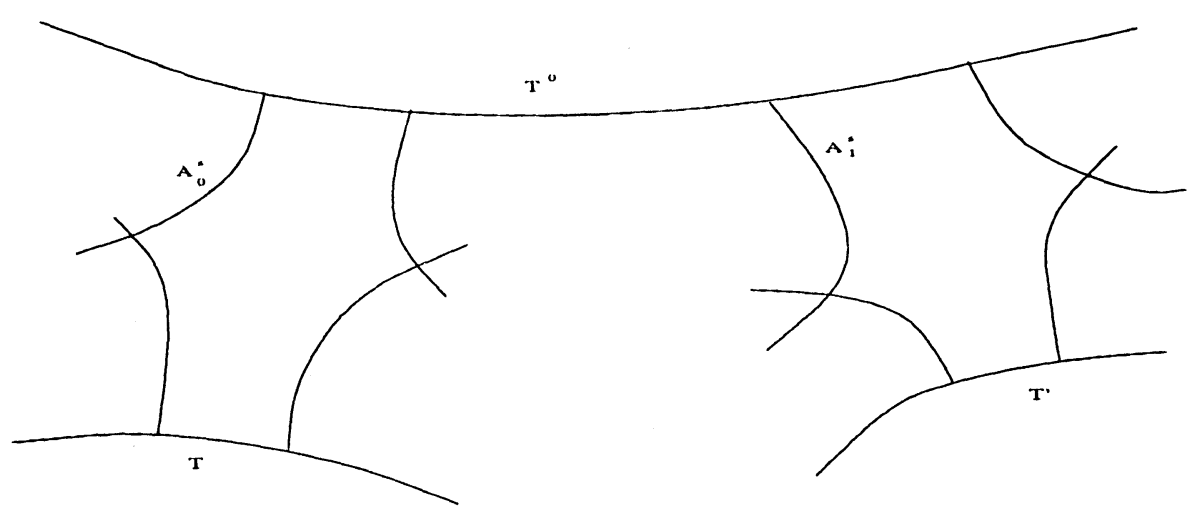

Figure 8: $\widetilde{A}_{1}^{s}\left(\right.$ resp. $\left.T^{\prime}\right)$ is "greater" than $\widetilde{A}_{0}^{s}($ resp. $T$ ).

Let $\widetilde{T}$ and $\widetilde{T}^{\prime}$ be two elements of $\mathcal{T}$ such that $\widetilde{T}^{0} \vdash_{1} \widetilde{T}$ and $\widetilde{T}^{0} \vdash_{1} \widetilde{T}^{\prime}$. Then, if $\widetilde{T}^{\prime}$ is greater than $\widetilde{T}$ in the meaning defined above, we will denote by $\widetilde{T} \vdash_{\widetilde{T}^{0}}^{s} \widetilde{T}^{\prime}$. More generally, for every pair $\left(\widetilde{T}, \widetilde{T}^{\prime}\right)$ of elements of $\mathcal{T}$ we will note $\widetilde{T} \vdash^{s} \widetilde{T}^{\prime}$ if for some element $\gamma$ of $\Gamma$ we have $\gamma \widetilde{T} \vdash_{\widetilde{T}^{0}}^{s} \gamma \widetilde{T}^{\prime}$. It gives an local order on $\widehat{\mathcal{D}}_{\infty}$ : we will denote by $w \ll^{s} w^{\prime}$ if the two elements $w$ and $w^{\prime}$ of $\overline{\mathcal{D}}_{\infty}$ admit representatives $\left(\widetilde{T}_{i}\right)_{i \in}$ and $\left(\widetilde{T}_{i}^{\prime}\right)_{i \in}$ such that:

- $\widetilde{T}_{0}=\widetilde{T}_{0}^{\prime}$

- $\widetilde{T}_{1} \vdash^{s} \widetilde{T}_{1}^{\prime}$

Note that two elements of $\widehat{\mathcal{D}}_{\infty}$ which are not $\mathcal{R}^{s}$-equivalent are comparable if and only if the sequences representing them admit a common occurence of the same element of $\mathcal{T}$. The relation $\ll^{s}$ is $\Gamma$-invariant.

Proposition 9.9. Let $x$ and $y$ be two different elements of $\mathcal{D}_{\infty}^{\Phi}$. Then, we have $\bar{I}_{\infty}(x) \ll^{s} \bar{I}_{\infty}(y)$ if and only if $x$ and $y$ belong to the same $\gamma \Omega$ for some element $\gamma$ of $\Gamma$ and that $s(x) \prec^{s} s(y)$.

In a similar way we can define a local order $\ll^{u}$ on $\widehat{\mathcal{D}}_{\infty}$ cooresponding to the order $\prec^{u}$ on $Q^{u}$. We are ready now for the proof of the main theorem of this section: 
Theorem 9.10. Two non $\mathbf{R}$-covered Anosov flows on the same $B L$ manifold are topologically equivalent.

Proof. Let $\Phi^{t}$ and $\Psi^{t}$ be two non $\mathbf{R}$-covered Anosov flows on the same BLmanifold $M$. Let $Q^{\Phi}$ and $Q^{\Psi}$ be their orbit spaces. We note $\mathcal{G}_{\Phi}^{s}, \mathcal{G}_{\Phi}^{u}, \mathcal{G}_{\Psi}^{s}$ and $\mathcal{G}_{\Psi}^{u}$ the traces of the weak lifted foliations on $Q^{\Phi}$ and $Q^{\Psi}$. According to the proposition 8.5 $\Phi^{t}$ and $\Psi^{t}$ are BL-flows. After isotopy, we can assume that they have the same associated triple $\left(T, A^{s}, A^{u}\right)$. Let $\mathcal{D}_{\infty}^{\Phi}$ be the subset of $Q^{\Phi}$ defined above corresponding to the orbits of $\Phi^{t}$ meeting $T$ an infinite number of time. Let $\mathcal{D}_{\infty}^{\Psi}$ be the analog subset of $Q^{\Psi}$. The itinerary map induced a bijection $f$ between $\mathcal{D}_{\infty}^{\Phi}$ and $\mathcal{D}_{\infty}^{\Psi}$. This bijection satisfies the following properties:

- It commutes with the actions of the fundamental group $\Gamma$,

- It maps the restriction of $\left(\mathcal{G}_{\Phi}^{s}, \mathcal{G}_{\Phi}^{u}\right)$ on $\mathcal{D}_{\infty}^{\Phi}$ over the restriction of $\left(\mathcal{G}_{\Psi}^{s}, \mathcal{G}_{\Psi}^{u}\right)$ on $\mathcal{D}_{\infty}^{\Psi}$

- Finally, $f$ preserves the local order between the stable or unstable leaves.

Let $\theta_{0}$ be the intersection between $A^{s}$ and $A^{u}$. It is a periodic orbit for both $\Phi^{t}$ and $\Psi^{t}$. Let $\Theta_{0}=\pi^{-1}\left(\theta_{0}\right)$ be its preimage in $\widetilde{M}$. Finally, we define $Q_{*}^{\Phi}=Q^{\Phi} \backslash \pi^{\Phi}\left(\Theta_{0}\right)$ and $Q_{*}^{\Psi}=Q^{\Psi} \backslash \pi^{\Psi}\left(\Theta_{0}\right)$. We call a periodic markov rectangle an open subset $R$ of $Q_{*}^{\Phi}$ such that:

- $R$ is contained in some $\gamma \Omega \quad(\gamma \in \Gamma)$,

- there are two periodic points $x_{1}$ and $x_{2}$ in $\gamma \Omega$ such that $R$ is the intersection between $\mathcal{L}^{++}\left(x_{1}\right)$ and $\mathcal{L}^{--}\left(x_{2}\right)$ or the intersection between $\mathcal{L}^{+-}\left(x_{1}\right)$ and $\mathcal{L}^{-+}\left(x_{2}\right)$.

In the definition above, the terminology "periodic point" means that $x_{1}$ is a fixed point of some element of $\Gamma$, and that $x_{2}$ is a fixed point of another element of $\Gamma$. We denote by $R_{x_{1}, x_{2}}$ this periodic markov rectangle when we want to explicit its vertices. Note that a periodic point in $\gamma \Omega$ belongs necessarily to $\mathcal{D}_{\infty}^{\Phi}$.

We define the analog notion in $Q_{*}^{\Psi}$. Since $\Phi^{t}$ and $\Psi^{t}$ are topologically transitive, the periodic markov rectangles are basis of the topologies of $Q_{*}^{\Phi}$ and $Q_{*}^{\Psi}$.

Since $f$ preserves the foliations and the local orders in the leaf spaces, we have: 
Lemma 9.11. For every periodic markov rectangle $R$ in $Q^{\Phi}$ we have the equality

$$
f\left(\mathcal{D}_{\infty}^{\Phi} \cap R_{x_{1}, x_{2}}\right)=\mathcal{D}_{\infty}^{\Psi} \cap R_{f\left(x_{1}\right), f\left(x_{2}\right)} .
$$

Lemma 9.12. If the periodic markov rectangle $R$ is contained in the periodic markov rectangle $R^{\prime}$, then $f\left(R \cap \mathcal{D}_{\infty}^{\Phi}\right)$ is contained in $f\left(R^{\prime} \cap \mathcal{D}_{\infty}^{\Phi}\right)$.

We will be a little more explicit for the proof of the following lemma:

Lemma 9.13. Let $x$ be a point of $Q_{*}^{\Phi}$. Then, the intersection of the $R_{f\left(x_{1}\right), f\left(x_{2}\right)}$ when $R_{x_{1}, x_{2}}$ describes all the periodic markov rectangle containing $x$, contains one and only one element of $Q_{*}^{\Psi}$.

Proof. We denote by $Q^{s}(\Phi), Q^{u}(\Phi), Q^{s}(\Psi)$ and $Q^{u}(\Psi)$ the leaf spaces associated to $\Phi^{t}$ and $\Psi^{t}$. Since $\Phi^{t}$ is topologically transitive, for every pair $\left(R, R^{\prime}\right)$ of periodic markov rectangle containing $x$, there is another periodic markov rectangle containing $x$ whose closure is contained in the intersection $R \cap R^{\prime}$. Let $\left(R_{x_{1}^{n}, x_{2}^{n}}\right)_{n \in \mathrm{N}}$ be a decreasing sequence of periodic markov rectangles whose intersection is exactly $\{x\}$. Each of them defines two intervals $\left[s\left(x_{1}^{n}\right), s\left(x_{2}^{n}\right)\right]$ and $\left[u\left(x_{1}^{n}\right), u\left(x_{2}^{n}\right)\right]$ in $Q^{s}(\Phi)$ and $Q^{u}(\Phi) . \quad\left[s\left(f\left(x_{1}^{n}\right)\right), s\left(f\left(x_{2}^{n}\right)\right)\right]$ and $\left[u\left(f\left(x_{1}^{n}\right)\right), u\left(f\left(x_{2}^{n}\right)\right)\right]$ are strictly decreasing sequences of intervals in $Q^{s}(\Psi)$ and $Q^{u}(\Psi)$. Therefore, their intersections are intervals $J^{s}$ and $J^{u}$ of $Q^{s}(\Psi)$ and $Q^{u}(\Psi)$. The intersection of the $R_{f\left(x_{1}^{n}\right), f\left(x_{2}^{n}\right)}$ is homeomorphic to the product $J^{s} \times J^{u}$. If the interior of $J^{s}$ is not empty, then it would contain two different leaves $s(\bar{x})$ and $s\left(\bar{x}^{\prime}\right)$ such that $\bar{x}$ and $\bar{x}^{\prime}$ belongs both to $\mathcal{D}_{\infty}^{\Psi}$. Then $s\left(f^{-1}(\bar{x})\right)$ and $s\left(f^{-1}\left(\bar{x}^{\prime}\right)\right)$ would be two different elements of the intersection of all the $\left[s\left(x_{1}^{n}\right), s\left(x_{2}^{n}\right)\right]$. But this intersection is exactly $\{s(x)\}$ : contradiction. Therefore, $J^{s}$ contains a unique point. By the same way, we prove that $J^{u}$ contains a unique point. Therefore, the intersection between the $R_{f\left(x_{1}^{n}\right), f\left(x_{2}^{n}\right)}$ is a single point. This intersection point belongs to $Q_{*}^{\Psi}$ since a periodic markov rectangle cannot contain an element of $\pi^{\Psi}\left(\Theta_{0}\right)$.

If we take another decreasing sequence of periodic markov rectangles whose intersection is $\{x\}$, the intersection of this new sequence with the sequence $\left(R_{x_{1}^{n}, x_{2}^{n}}\right)_{n \in \mathrm{N}}$ is still a decreasing sequence of periodic markov rectangles. Hence the intersection does not depend on the initial choice of this sequence.

With the notation of the lemma 9.13 , we can define $f(x)$ as the unique element of the intersection of the $R_{f\left(x_{1}\right), f\left(x_{2}\right)}$. We obtain thus a map $f$ 
between $Q_{*}^{\Phi}$ and $Q_{*}^{\Psi}$. This map is bijective, commutes with the actions of $\Gamma$ and preserves the natural foliations. Furthermore, since it preserves the local orders in the leaf spaces, it is an homeomorphism. Since $\pi^{\Phi}\left(\Theta_{0}\right)$ and $\pi^{\Psi}\left(\Theta_{0}\right)$ are discrete subsets of $Q^{\Phi}$ and $Q^{\Psi}$, we can extend $f$ as a topological conjugacy between the actions of $\Gamma$ on $Q^{\Phi}$ and $Q^{\Psi}$. According to the theorem 1.1 the flows $\Phi^{t}$ and $\Psi^{t}$ are topologically equivalent (note that $f$ maps $\mathcal{G}_{\Phi}^{s}$ on $\mathcal{G}_{\Psi}^{s}$ ).

This theorem completes the proof of the second assertion of theorem B.

\section{References.}

[1] D.V. Anosov, Geodesic flows on closed riemannian manifolds with negative curvature, Proc. Steklov Inst. Math. AMS Translations (1969).

[2] T. Barbot, Géométrie transverse des flots d'Anosov, Thesis, Lyon (1992).

[3] T. Barbot, Caractérisation des flots d'Anosov en dimension 3 par leurs feuilletages faibles, Ergod. Th. \& Dynam. Sys., 15 (1995), 247-270.

[4] T. Barbot, Mise en position optimale de tores par rapport à un flot d'Anosov, Comment. Math. Helv., 70 (1995), 113-160.

[5] T. Barbot, Flots d'Anosov sur les variétés graphées au sens de Waldhausen, Ann. Inst. Fourier, Grenoble, 46 (1996), 1451-1517.

[6] C. Bonatti and R. Langevin, Un exemple de flot d'Anosov transitif transverse $\grave{a}$ un tore et non conjugué à une suspension, Ergod. Th. \& Dynam. Sys., 14 (1994), 633-643.

[7] R. Bowen, On Axiom A diffeomorphisms, A.M.S. Providence, 35 (1970).

[8] M. Brunella, Separating the basic sets of a nontransitive Anosov flow, Bull. London Math. Soc., 25 (1993), 487-490.

[9] J.P. Christy, Anosov flows on three-manifolds, Thesis, Berkeley (1984).

[10] S. Fenley, Quasigeodesic Anosov flows and homotopic properties of closed orbits, Jour. Diff. Geom., 41 (1995), 479-514.

[11] J. Franks, Anosov diffeomorphisms, Global Analysis, Proc. Symp. Pure Math., A.M.S., XIV (1970), 61-93.

[12] D. Fried, Transitive Anosov flows and pseudo-anosov maps, Topology, 22 (1983), 299-304. 
[13] E. Ghys, Flots d'Anosov sur les 3-variétés fibrées en cercles, Ergod. Th. \& Dynam. Sys., 4 (1984), 67-80.

[14] E. Ghys, Déformations de flots d'Anosov et de groupes fuchsiens, Ann. Inst. Fourier, Grenoble 42 (1992), 209-247.

[15] S. Goodman, Dehn surgery on Anosov flows, SLN 1007, Springer, New York (1983).

[16] M. Handel and W. Thurston, Anosov flows on new 3-manifolds, Invent. Math., 59 (1980), 95-103.

[17] M. Hirsch and C. Pugh, Stable manifolds and hyperbolic sets, Proc. Symp. Pure Math., A.M.S., XIV (1970).

[18] S. Matsumoto, Codimension one foliations on solvable manifolds, Comment. Math. Helv., 68 (1993), 633-652.

[19] J. Morgan and P. Shalen, Valuation trees and degeneration of hyperbolic structures I, Ann. of Math., 122 (1985), 398-476.

[20] R. Moussu, Sur les feuilletages de codimension un, Thesis, Orsay (1971).

[21] S. Newhouse, On codimension one Anosov diffeomorphisms, Amer. J. Math. 92 (1970).

[22] J.F. Plante, Anosov flows, Amer. Jour. Math., 94 (1972), 729-754.

[23] J.F. Plante, Anosov flows, transversely affine foliations and a conjecture of Verjovsky, J. London Math. Soc., 23 (1981), 358-362.

[24] C. Pugh and M. Shub, The $\Omega$-stability theorem for flows, Invent. Math., 11 (1970), 150-158.

[25] S. Smale, Differentiable dynamical systems, Bull. Amer. Math. Soc., 73 (1967), 747-817.

[26] J. Tits, A "theorem of Lie-Kolchin" for trees, Contribution to Alg., Academic Press (1977), 377-388.

[27] P. Tomter, Anosov flows on infra-homogeneous spaces, Global Analysis, Proc. Symp. Pure Math., A.M.S., XIV (1970), 299-327.

[28] F. Waldhausen, Eine Klasse von 3-dimensionalen Mannigfaltigkeiten. I, Invent. Math., 3 (1967), 308-333.

[29] F. Waldhausen, Eine Klasse von 3-dimensionalen Mannigfaltigkeiten. II, Invent. Math., 4 (1967), 87-117. 
Received OCtober 16, 1996.

ENS LYON

Departement de Mathematiques

46 ALLEE D'ITALIE

69364 LYON CEDEX 7

FRANCE

E-MAIL: BARBOT@UMPA.ENS-LYON.FR 